\title{
Clustering of health behaviours in Canadi- ans: A multiple behaviour analysis of data from the Canadian Longitudinal Study on Aging
}

\section{Zack van Allen ${ }^{1,2}$, Simon L. Bacon ${ }^{3,4}$, Paquito Bernard ${ }^{5,6}$, Heather Brown $^{7}$, Sophie Desroches ${ }^{8}$, Monika Kastner ${ }^{9}$, Kim Lavoie ${ }^{4}$, Marta Marques $^{10}$, Nicola McCleary ${ }^{1,2}$, Sharon Straus', Monica Taljaard ${ }^{1,2}$, Kednapa Thavorn ${ }^{1,2}$, Jennifer R. Tomasone ${ }^{1,}$, Justin Presseau ${ }^{1,2^{*}}$}

\begin{abstract}
Health risk behaviours such as physical inactivity, unhealthy eating, smoking tobacco, and alcohol use are each leading risk factors for non-communicable chronic disease and each play a central role in limiting health and life satisfaction. However, much less is known about how co-occurring behaviours are associated with health outcomes. Understanding which behaviours tend to co-occur (i.e., cluster together), and how such clusters are associated with physical and mental health, life satisfaction, and health care utilization may provide novel opportunities to leverage this co-occurrence to develop and evaluate interventions to promote multiple health behaviour change. Using cross-sectional baseline data $(\mathrm{N}=40,268)$ from the Canadian Longitudinal Study of Aging, we performed a pre-defined set of analyses to examine the co-occurrence of health behaviours. We used agglomerative hierarchical cluster analysis to cluster individuals based on their behavioural tendencies and multinomial logistic regression to examine how these clusters are associated with demographic characteristics, healthcare utilization, and general health and life satisfaction, and assess whether sex and age moderate these relationships. Seven clusters were identified with clusters differentiated by six of the seven health behaviours included in the analysis. Variability between clusters was observed in frequencies of weekly walking, strenuous exercise, and alcohol consumption. Sociodemographic characteristics varied across several clusters while selfreported physical/mental health showed less variation across clusters. The seven identified clusters of health behaviours allow for contrasts to be made with comparable analyses in other countries and will help inform the development of future health behaviour change interventions tailored to sub-populations and their sociodemographic profiles.
\end{abstract}

\section{Keywords}

health behaviours, multiple behaviours, cluster analysis, network analysis, CLSA

\footnotetext{
IOttawa Hospital Research Institute, Ottawa, ON, Canada; $\quad$ 2University of Ottawa, Ottawa, ON, Canada; $\quad 3$ Concordia University, Montreal, QC, Canada; 4 Montreal Behavioural Medicine Centre, CIUSSS-NIM, Montreal, QC, Canada; $\quad$ 5University of Quebec in Montreal, Montreal, QC, Canada; 6 Research Center of the Montreal Mental Health University Institute, Montreal, QC, Canada; 7 Newcastle University, Newcastle upon Tyne, UK; $\quad$ 8Laval University, Quebec City, QC, Canada; $\quad$ 9University of Toronto, Toronto, ON, Canada; IOADAPT SFI Research Centre, Trinity College Dublin, Ireland IIQueens University, Kingston, ON, Canada
}

Corresponding Author:

Justin Presseau, jpresseau@ohri.ca. 
Non-communicable chronic diseases such as chronic respiratory disease, diabetes, cardiovascular disease, and cancer cause two thirds of annual deaths in Canada (Public Health Agency of Canada, 2016). Furthermore, nearly 12\% of people aged 65 or older have lived with two or more chronic conditions during their lifetime (Public Health Agency of Canada, 2017). Health risk behaviours such as smoking, excessive alcohol consumption, physical inactivity, and unhealthy eating are strongly associated with quality of life and are leading risk factors for chronic diseases (Fisher et al., 2011). With approximately four in five adult Canadians engaging in at least one of the health impacting behaviours associated with non-communicable chronic diseases, the prevalence of risky health behaviours is high (Public Health Agency of Canada, 2017).

The consequences and risk factors of multimorbidity (living with 2 or more chronic conditions) has been studied extensively (Marengoni et al., 2011; Nunes et al., 2016; Prados-Torres et al., 2014); however, research seeking to understand the relationships between life satisfaction, general health, and different combinations of health behaviours has received comparable little attention. Our daily lives are characterized by multiple co-occurring social, personal, family, health, and work-related behaviours, each contesting for the limited energy, motivation, and time available (Presseau et al., 2013). Despite this, health risk behaviours are generally promoted and studied in isolation resulting in interventions and guidelines for healthy living siloed by individual behaviours. For example, historically Canada has had separate guidelines for alcohol consumption (Butt et al., 2011), alcohol consumption (Canadian Center on Substance Abuse and Addition, 2018), and physical activity and sleep (Tremblay et al., 2011), although recent guidelines are beginning to incorporate multiple health behaviours (e.g., guidelines for movement behaviours; Ross et al., 2020) . The movement towards including multiple behaviour guidelines reflect a more realistic understanding of which health behaviours co-occur, and how these co-occurances are associated with health care utilization, life satisfaction, physical health, and mental health may provide new opportunities to promote multiple health behaviour change. Ideally, interventions should be tailored to reflect the real-world complexities of health risk behaviours through an understanding of which behaviours co-occur and for whom.

The co-occurrence, or clustering, of health behaviours has been demonstrated with international data. For example, Conry et al (2011) investigated the clustering of alcohol use, physical activity, smoking, and unhealthy eating in a sample of Irish adults obtained from the 2007 National Survey of Lifestyle, Attitudes, and Nutrition. Six clusters were identified in this crosssectional analysis which were labelled as: 1) 'multiple risk factor' (moderate physical activity, moderate to high alcohol use, variable healthy eating); 2) 'mixed lifestyle' (those who had never smoked, reported moderate physical activity, and variable alcohol consumption); 3) 'physically inactive' (people with low levels of physical activity, poor eating, who reporting some smoking and high alcohol use); 4) 'temperate' (moderately active and moderate drinkers who had never smoked); 5) 'former smokers' (former smokers who reported high physical activity, moderate alcohol use, and healthy eating); and 6) 'healthy lifestyle'(characterized by people who had never smoked, high physical activity, highest healthy eating, moderate alcohol use).

In a separate study, Buck and Frosini (2012) examined the clustering of unhealthy eating, alcohol use, smoking, and physical inactivity among adults aged 16-74 using 2003-2008 data from the Health Survey of England. Findings indicated that in 2008, 63\% of the sample engaged in one or two unhealthy behaviours, $25 \%$ engaged in three or more risky health behaviours, and $5 \%$ reported engaging in all four measured health behaviours. Only $7 \%$ of the sample did not engage in any measured risky health behaviours. Common co-occurring behaviours included inactivity and unhealthy eating (more prevalent in men), and unhealthy eating with physical activity (more prevalent in women) or alcohol use. When compared to women, men were more likely to report three co-occurring unhealthy behaviours and respondents aged $65+$ were more likely than any other age group to engage in two unhealthy behaviours.

Although the previous examples have investigated co-occurring behaviours in the general public, some studies have extended this work to focus on adults aged 50 years and older who engage in risky health behaviours less frequently than younger adults (e.g., Noble et al., 2015). One study using cross sectional data from Germany collected from respondents aged 50-70 and studied the clustering of four health risk behaviours (physical inactivity, unhealthy eating, alcohol use, and tobacco use). Individuals were grouped into five clusters based on the 
frequencies of their health behaviours. Twenty five percent of the sample did not engage in any unhealthy behaviours while $21 \%$ were inactive, $13 \%$ were smokers with other risk behaviours, $18 \%$ ate low amounts of fruits and vegetables, and $23 \%$ were described as drinkers with other risk behaviours (Schneider et al., 2009). Interestingly, while some people's behaviours were characterized by engaging in one unhealthy behaviour, more than a third engaged in multiple unhealthy behaviours which clustered together. Additionally, cluster membership was also associated with sociodemographic factors with people engaging in multiple unhealthy behaviours more likely to be men, report lower socio economic status, and live alone. Unfortunately, variability in measurement tools and a lack of cluster naming conventions in multiple health behaviour cluster studies impedes direct comparisons between clusters identified in different countries to date.

Finally, Shaw and Agahi (2012) employed a different methodological approach called 'health behaviour profiles' to assess co-occurring health risk behaviours in adults 50 years or older using baseline data from the Health and Retirement study (Heeringa \& Conner, 1995). Overall, twelve health behaviour profiles were created using all combinations of physically active vs inactive, smokers vs non-smokers, and those who reported no vs moderate vs heavy alcohol consumption. The percentage of people represented in each profile varied widely with the six most prevalent profiles including: 1) 'physically active, non-drinkers, who smoke' (4.2\%); 2) 'physically inactive, non-drinkers, who smoke' (6.5\%); 3) 'physically inactive, moderate drinkers, who do not smoke' (8.6\%); 4) 'physically active, moderate drinkers, who do not smoke' (10.1\%); 5) 'physically active, non-drinkers, who do not smoker (23.7\%); and 6) 'physically inactive, non-drinkers, who do not smoke' (34.1\%).

Cumulatively, survey data from multiple nations has shown that health risk behaviours cluster differently across the population and that these clusters are associated with distinct health outcomes and sociodemographic patterns. However, limited research has explored health behaviour co-occurrence with Canadian data, with Canadian National Population Survey data demonstrating that smoking, alcohol use, and physical inactivity co-occur (deRuiter et al., 2014, 2016). The Canadian context is unique with an ethnically diverse population, relatively high smoking prevalence, and unique challenges to some health behaviours (e.g., seasonality is a barrier to physical activity in the winter) A clearer understanding of which behaviours tend to cluster together at the population level may provide novel insights to support and guide clinicians, policy makers, and patients in preventing non-communicable diseases using a multiple behaviour approach. Additionally, the clustering of health behaviours has the potential to inform future guideline development (e.g., Ross et al., 2020). For example, guidelines for smoking cessation may acknowledge that physical inactivity and alcohol use often cooccurs with smoking behaviour and provide recommendations for multiple behavioural changes.

To this end, we analyzed cross-sectional baseline data from the Canadian Longitudinal Study on Aging (CLSA; Raina et al., 2009), representing the most publicly available CLSA dataset at the time of this study, to identify patterns of co-occurring behaviours and assess how sociodemographic and health indicators are associated with these patterns.

\section{METHODS}

Detailed methodological information is shown in the published protocol (van Allen et al., 2021) which is summarized here in brief. The Canadian Longitudinal Study of Aging (CLSA) is a longitudinal, nationally representative, study designed to measure societal, biological, physical, and psychosocial factors related to healthy aging (Raina et al., 2009). Baseline data collection for the CLSA was collected between 2010-2015 comprising two approaches. First, the 'tracking' cohort $(\mathrm{n}=21,241)$ completed data collected via an hour-long computer assisted phone interviews. Second, the 'comprehensive' cohort $(n=30,097)$ completed an in-person interview lasting 90-minutes as well as a data collection site visit. Additionally, a 'maintaining contact questionnaire' was administered over the phone for the comprehensive and tracking cohorts. The maintaining contact questionnaire, tracking cohort, and comprehensive cohort form the baseline data collected used in this analysis. Data are available from the Canadian 
Longitudinal Study on Aging (www.clsa-elcv.ca) for researchers who meet the criteria for access to de-identified CLSA data.

\section{Participants}

Participants were recruited through random-digit dialing, provincial health registries, and the Canadian Community Health Survey on Healthy Aging (Raina et al., 2009; Wolfson et al., 2009). Exclusion criteria for the CLSA included: residents living in three territories and First Nations reserves, full time members of the Canadian Armed Forces, people living with cognitive impairments, and individuals living in institutions (including 24-hour nursing homes) ( Raina et al., 2008). Participants included in the study included $n=51,338$ French and Englishspeaking Canadians (51\% female) between the ages of 45-85 at time of enrollment. The average participant age is 62.98 years old $(S D=10.43$ ) with $26 \%$ between $45-54$ years, $32 \%$ between $55-64$ years, $23 \%$ between $65-74$ years, and $18 \%$ between $75-85$ years. A full description of demographic characteristics of the sample, as well as summary data across all measured variables is available in the CLSA baseline data report (Raina et al., 2018).

Variable Selection. The CLSA baseline dataset contains approximately 1800 variables. One of the most important decisions in cluster analysis is selecting a parsimonious set of variables, or features, to include in the model. Variable selection can be performed by objective or subjective approaches. Our approach is the latter. Objective methods rely on data-driven techniques (e.g., forward/backward selection) and techniques such as factor analysis and principal components analysis for dimension reduction (Deliu et al., 2016). Subjective approaches are generally driven by expert opinion and/or theory-driven research questions. Based on our research objectives, and the data collected by CLSA, we identified an initial set of variables assessing health behaviours, non-health behaviours, sociodemographic indicators, general health and well-being, and health care service utilization. Our decisions were also shaped by issues of survey design (e.g., skip-questions), knowledge of basic summary statistics for baseline CLSA data (P. Raina et al., 2018), and our own supplementary summary statistics on the baseline data ${ }^{1}$. See Appendix 1 for a description of each variable to be used in analyses and example items.

Health Behaviours. Physical activity and sedentary behaviour were measured as independent behaviours with the Physical Activity Scale for the Elderly (PASE; Washburn et al., 1993) which assesses the frequency of sedentary behaviour, walking, light physical activity, moderate physical activity, strenuous physical activity, and exercise. Items asked participants to report on their activity levels over the previous 7 days on a 1 (never) to 4 (often, 5-7 days) scale. A recent report published by Statistics Canada focusing on the relationship between physical activity and lung functioning (Dogra et al., 2019) merged light and moderate physical activity together and also merged strenuous physical activity and exercise together based on issues with question prompts and conceptual overlap between question items. To facilitate dimension reduction, we opted for a similar approach in which the PASE subscale items were merged to represent: sitting, walking, light/moderate physical activity (renamed 'light sports' to avoid confusion with 'light-to-moderate physical activity'; e.g., Saint-Maurice et al., 2018), and strenuous physical activity/ exercise. Fruit and vegetable consumption was assessed with one item from the Seniors in the Community Risk Evaluation for Eating and Nutrition questionnaire (Keller et al., 2005). The item asks respondents how many servings of fruits and vegetables they eat in a day. The original scale was scored 1 (seven or more) to 7 (less than two); however, items will be reverse coded such that higher scores indicate more fruit and vegetable consumption. Smoking behaviour was measured using a skip-question framework in the CLSA. Participants who answered 'no' to the question 'have you smoked at least 100 cigarettes in your life' and responded 'yes' to the question 'have you ever smoked a whole cigarette' were subsequently asked whether they smoke occasionally, daily, or not at all in the past 30 days. Next, only participants who reported smoking occasionally or daily were asked follow-up

1 The research team received access to the CLSA datasets prior to the publication of the protocol. We computed means and standard deviations for the variables used in this study in order to inform our analytical choices. None of the analyses were conducted prior to submission of the protocol. 
questions pertaining to frequency and types of tobacco products used. Descriptive analysis on these last two items showed that only a small minority of respondents $(n=4,845 ; 9.4 \%)$ engaged in occasional or daily smoking with the majority $(n=30,558 ; 59.5 \%)$ not engaging in smoking behaviour in the past 30 days. ${ }^{2}$ Although frequency of smoking would be a more informative metric, any cluster analysis with smoking frequency would reduce the sample size to $n=4,845(9.4 \%)$ and would only represent people who have smoked within the past 30 days. Additionally, we assigned a value of 0 to each respondent who responded 'no' to the question 'have you ever smoked a whole cigarette' as these individuals also did not smoke in the past 30 days. A similar approach has been applied to skip structure data when missing data represent the absence of a behaviour or psychological feature (Borsboom \& Cramer, 2013). Ultimately, this creates three levels distinguishing between people who smoked occasionally, daily, or not at all during a 30-day window. Alcohol use was assessed with a single item asking participants how often they drank alcohol in the past 12 months on a scale from 1 (almost every day) to 7 (less than once a week). Responses were reverse coded so higher values indicate greater alcohol consumption. Finally, sleep was also measured with a single item. Participants were asked how many hours of sleep they get, on average, during the past month and could respond with any value between $0-24$. This variable was originally included in the analysis plan (van Allen et al., 2020) but was subsequently removed due to high (41.5\%) missingness of the data.

Sociodemographic Indicators. We used several sociodemographic indicators in our analysis. These include age, as grouped in the CLSA dataset (45-54; 55-64; 65-74; 75-85), sex (male/female), marital status (single, married or common-law, widowed, divorced, separated), household income ( $<20 \mathrm{k}, \$ 20-\$ 49 \mathrm{k}, \$ 50-\$ 99 \mathrm{k}, \$ 100-\$ 149 \mathrm{k}, \$ 150 \mathrm{k}+)$, retirement status (completely retired, partly retired, not retired), and working status (yes/no to 'are you currently working at a job or business'). Additionally, participants responded to 19 questions from the Medical Outcomes Study (MOS) Social Support Survey (Sherbourne \& Stewart, 1991). The MOS is scored according to 5 subscales: tangible social support, affection, positive social interaction, and emotional and informational support. A MOS 'overall support index' is also scored in the CLSA baseline dataset. To reduce the number of constructs in our analyses, we used the overall support index, scored from 0 (low support) to 100 (high support).

General Health and Life Satisfaction. Three single item measures were selected from the CLSAs general health module. These include an indicator of general health ('in general, would you say your health is excellent, very good, good, fair, or poor?'), mental health ('in general, would you say your mental health is excellent, very good, good, fair, or poor?'), and perceptions of healthy aging (in terms of your own healthy aging, would you say it is excellent, very good, good, fair, or poor?'). Items were originally scored on a 1 (excellent) to 5 (poor) but will be reverse coded. Additionally, a composite score from the satisfaction with life questionnaire (SWLS; Diener et al., 1985) was used in analysis. The SWLS is scored according to Deiner ( Diener, 2006) on a scale ranging from 1 (extremely dissatisfied) to 7 (extremely satisfied). Finally, a measure of body mass index (BMI) was used as an indicator of physical health.

Health Care Utilization. Three single item questions were selected from the CLSA's health care utilization module. These items represent emergency department visits ("Have you been seen in an Emergency Department during the past 12 months?"), hospital admittance ("Were you a patient in a hospital overnight during the past 12 months?"), and nursing home usage ("Were you a patient in a nursing home or convalescent home during the past 12 months?"). All responses were coded yes (1) or no (0).

\section{Cluster Analysis Overview}

Classifying data through the assignment of classes to objects in a dataset is a common application of machine learning (i.e., "set of methods that can automatically detect patterns in data, and then use the uncovered patterns to predict future data" (Murphy, 2012).

\footnotetext{
2 Many participants were not presented with this question $(n=15,823 ; 30.8 \%)$. The remaining respondents $(n=$ $112 ; 0.2 \%$ ) opted not to respond to the question.
} 
Classification algorithms fall into three categories: supervised learning, semi-supervised learning, and unsupervised learning. In supervised learning, the relationship between the input and target variables are known. An algorithm is 'supervised' in that it can be trained on a dataset that contains correct classifications. Datasets containing these correct classifications are referred to as 'labeled data' in contrast to 'unlabeled data' in which the correct classifications are not known. In semi-supervised learning a combination of labeled and unlabeled data is used to model the data, while in unsupervised learning the model works on its own do discover patterns in unlabeled data (Fung, 2001).

Cluster analysis is a type of unsupervised machine learning that comprises a set of methods for identifying distinct characteristics in heterogenous samples and clustering them into homogenous groups (Rapkin \& Luke, 1993). When the target number of clusters $(k)$ is known, partitioning based clustering arguments such as $k$-means, $k$-medoids, or model-based clustering approaches are appropriate. However, when $k$ is unknown, as is the case with clusters of Canadians based on health and non-health behaviours, hierarchical clustering is a suitable method (Rapkin \& Luke, 1993).

The hierarchical structure of the data can be obtained through clustering individual data points in a bottom-up approach (i.e., agglomerative clustering) or by partitioning a single cluster into smaller clusters until each cluster is a single observation through a top-down approach (i.e., divisive clustering). Divisive methods are rarely used in practice due to heavy computational requirements (Xu \& Wunsch, 2005). In agglomerative hierarchical clustering, each individual data point is initially treated as its own cluster. The methodological process is as follows (Alashwal et al., 2019): 1) each data point is assigned to its own cluster; 2) the distance between each cluster is calculated; 3 ) the pair of clusters with the shortest distance between them is selected and merged into a single cluster; 4) the distances between the new cluster and all other clusters are recalculated; and 5) these steps are repeated until only one cluster remains. However, a single cluster $(k=1)$ is unlikely to be informative; researchers can identify the number of clusters that best describe the data (e.g., $k=5)$ through subjective criteria and/or with the aid of statistical tests that have been developed for this purpose (see methods section below).

Several measures of 'distance' are widely used in practice, though the Gower distance (Gower, 1971) is appropriate for mixed data (binary, ordinal, continuous). In addition to selecting a measure of distance, hierarchical agglomerative clustering also requires a linkage method to be specified to define how the distance between clusters is calculated. Different methods exist for specifying the anchor points used to calculate the distance between clusters (i.e., how the distances between clusters are 'linked'). For example, 'single/minimum linkage' calculates the minimum distance between data points in each cluster while 'centroid linkage' calculates the distance between the center of each cluster (Fung, 2001). No consensus exists as to which linkage method is superior, though it is recognised that final clustering solutions may differ based on the linkage method selected (Xu \& Wunsch, 2005).

\section{Cluster Analysis}

\section{RESULTS}

Prior to performing cluster analysis, all health behaviour variables (walking, sitting, light sports, exercise, smoking, alcohol) were standardized using the scale function in base R (RC Team, 2019). Listwise deletion was applied to missing data in health behaviour variables resulting in a total sample of $n=40,268$. We then performed hierarchical agglomerative cluster analysis with five linkage methods (e.g., complete-linkage, single-linkage, average-linkage, centroid-linkage, and Ward's method) using 'hclust' function supported by the package 'fastcluster' (Müllner, 2013) to optimize performance. Gower distance was computed using the 'daisy' function in the 'cluster' package (Maechler et al., 2012).

We examined the outputs of these cluster analyses by looking at summary statistics for health behaviour variables for each linkage method with four-clusters each. Two of the five linkage methods produced interpretable and useful clustering solutions (Ward and completelinkage) while the other methods resulted in clusters with nearly all participants forming a single cluster with a small number of participants (often one per cluster) forming the remaining groups. Next, we employed a data-driven approach to determine the optimal number of clusters using complete linkage and Ward's method. We used the NbClust package (Charrad et 
al., 2014) to provide the top three clustering solutions for both linkage methods. This resulted in six options for combinations of linkage measures and $k$. The research team opted for a clustering solution with 7 clusters using Ward's method for linkage (for full analysis see online appendix).

\section{Descriptive Analysis of Clusters}

Standardized means and standard deviations for health behaviours in each cluster are presented in Table 1. Demographic information for each cluster is presented in Table 2. Ridge plots illustrating the density distributions of responses for each health behaviour across clusters are presented in Figure 1. Descriptive summaries highlighting the characteristics of the final sample and each cluster, interpreted using unstandardized scales, are provided below and are accompanied by radar charts using standardized scales in Figure 2.

Final sample. Following listwise deletion for missing values in health behaviour variables there were 40,268 people included in the final sample. On average, people engaged in walking activities 3-4 days a week $(\mathrm{M}=3.1$; $\mathrm{SD}=1.1)$, sitting activities close to $4-5$ days a week $(\mathrm{M}=$ 3.9; $\mathrm{SD}=0.4$ ), light sports were mostly performed between 'never' and 'seldom (1-2 days per week; $\mathrm{M}=1.3 ; \mathrm{SD}=0.5$ ), people engaged in strenuous exercise approximately 1 day per week $(M=1.6 ; S D=.8)$, ate 4 servings of fruits and vegetables per day $(M=4.0 ; S D=1.8)$, were nonsmokers $(\mathrm{M}=0.9 ; \mathrm{SD}=0.8)$, and consumed alcohol near the middle of a 7 point scale ranging from monthly to daily $(M=4.2 ; S D=2.0)$. There were no sex differences in the overall sample $(50.2 \%$ Female). The majority were in married or common law relationships $(70.8 \%)$ with household incomes between $\$ 50.000-\$ 99,000$ per year $(35.6 \%)$. The distribution of age groups was 27.1\% (age 45-54), 32.8\% (age 55-64), 23.5\% (age 65-74), and 16.6\% (age 7585).

Table I. Standardized means and standard deviations for health behaviours and clusters

\begin{tabular}{|c|c|c|c|c|c|c|c|c|c|c|c|c|c|c|c|}
\hline \multirow[b]{2}{*}{ Cluster } & \multirow[b]{2}{*}{$\mathbf{n}$} & \multicolumn{2}{|c|}{ Walking } & \multicolumn{2}{|c|}{ Sitting } & \multicolumn{2}{|c|}{ Light Sports } & \multicolumn{2}{|c|}{ Exercise } & \multicolumn{2}{|c|}{ Fruit/Veg } & \multicolumn{2}{|c|}{ Smoking } & \multicolumn{2}{|c|}{ Alcohol } \\
\hline & & M & SD & $\mathbf{M}$ & SD & M & SD & M & SD & M & SD & $\mathbf{M}$ & SD & M & SD \\
\hline 1 & 7703 & 0.81 & 0.18 & 0.17 & 0.53 & 0.38 & 1.28 & 1.09 & 0.94 & 0.37 & 0.96 & -0.17 & 0.62 & 0.03 & 0.95 \\
\hline 2 & 7223 & 0.62 & 0.38 & 0.24 & 0.25 & -0.25 & 0.71 & -0.52 & 0.49 & -0.12 & 0.99 & -0.42 & 0.68 & -0.77 & 0.77 \\
\hline 3 & 4094 & -1.53 & 0.41 & 0.22 & 0.34 & -0.19 & 0.83 & -0.33 & 0.82 & -0.35 & 0.93 & -0.38 & 0.68 & -0.97 & 0.75 \\
\hline 4 & 10723 & -0.80 & 0.69 & 0.23 & 0.32 & 0.02 & 1.00 & 0.07 & 1.01 & 0.05 & 0.99 & -0.15 & 0.60 & 0.51 & 0.69 \\
\hline 5 & 5212 & 0.83 & 0.11 & 0.20 & 0.47 & 0.04 & 0.96 & -0.55 & 0.40 & 0.15 & 0.95 & -0.03 & 0.65 & 0.86 & 0.48 \\
\hline 6 & 3079 & -0.15 & 1.05 & -0.04 & 1.07 & -0.16 & 0.87 & -0.33 & 0.80 & -0.56 & 0.93 & 2.68 & 0.42 & -0.08 & 1.07 \\
\hline 7 & 2234 & 0.08 & 0.91 & -3.32 & 1.52 & -0.11 & 0.84 & -0.05 & 0.90 & -0.05 & 0.95 & -0.26 & 0.65 & -0.14 & 0.96 \\
\hline Full Sample & 40268 & 0.00 & 1.00 & 0.00 & 1.00 & 0.00 & 1.00 & 0.00 & 1.00 & 0.00 & 1.00 & 0.00 & 1.00 & 0.00 & 1.00 \\
\hline $\begin{array}{l}\text { Notes: Standardi } \\
\text { Cluster } 1 \text { = Phys } \\
\text { Walking, Light S } \\
\text { Alcohol Use; Clu }\end{array}$ & $\begin{array}{l}\text { Active He } \\
\text { and Exer } \\
=\text { Occasi }\end{array}$ & $\begin{array}{l}\text {; Clus } \\
1 \text { and }\end{array}$ & $\begin{array}{l}\mathrm{r} 4=\mathrm{Fr} \\
\text { ily Smo }\end{array}$ & $\begin{array}{l}=\text { Freq } \\
\text { ent Alc } \\
\text { s who }\end{array}$ & $\begin{array}{l}\text { I Use : } \\
\text { equent }\end{array}$ & $\begin{array}{l}\text { with In } \\
\text { Infrequ } \\
\text { Eat Fruit }\end{array}$ & $\begin{array}{l}\text { Ient S } \\
\text { Walke } \\
\text { d Veg }\end{array}$ & $\begin{array}{l}\text { Cluster } \\
\text { les anc }\end{array}$ & $\begin{array}{l}\text { ise ar } \\
\text { Frequ } \\
\text { rcise }\end{array}$ & $\begin{array}{l}\text { dative } \\
\text { freque } \\
\text { Walke } \\
\text { ster } 7\end{array}$ & $\begin{array}{l}\text { mean } \\
\text { Alcohs } \\
\text { ith In } \\
\text { frequ }\end{array}$ & $\begin{array}{l}\text { ferences. } \\
\text { se; Clust } \\
\text { quent Str } \\
\text { Sedentar }\end{array}$ & $\begin{array}{l}\text { Cluster } \\
3=\text { In } \\
\text { nuous } \mathrm{B} \\
\text { Activit }\end{array}$ & $\begin{array}{l}\text { are as } \\
\text { tent Al } \\
\text { ise but }\end{array}$ & $\begin{array}{l}\text { lows: } \\
\text { ol Use, } \\
\text { her }\end{array}$ \\
\hline
\end{tabular}


Figure 2. Health behaviour ridge (density) plots clusters across clusters and final sample.
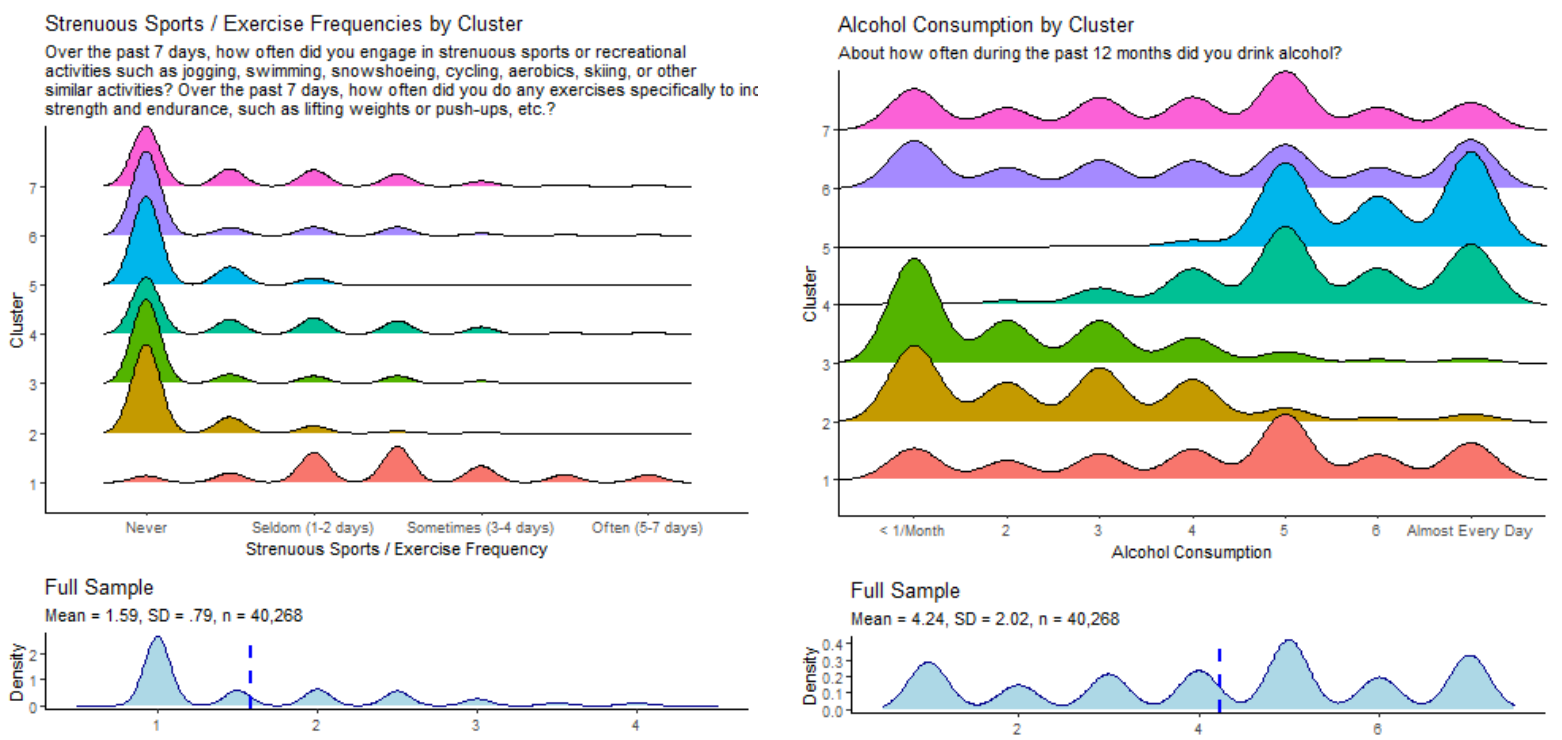

Full Sample

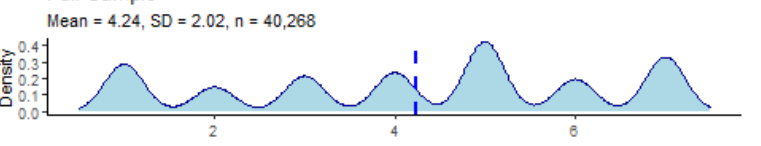

Light Sports Frequencies by Cluster

Over the past 7 days, how often did you engage in light sports or recreational activities such as bowling, golf with a cart, shuffleboard, badminton, fishing or other similar activities such as ballroom dancing, hunting, skating, golf without a cart, softball or other similar activities?

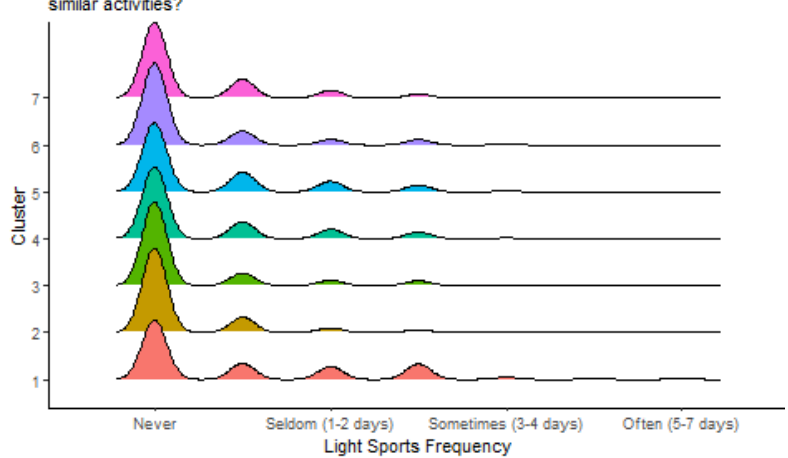

Smoking Frequency by Cluster

Have you ever smoked a whole cigarette? At the present time,

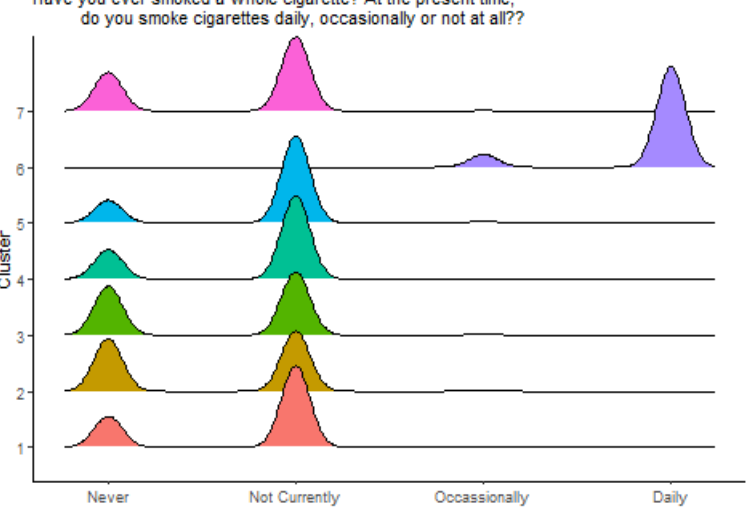

Full Sample

Mean $=1.29, \mathrm{SD}=.51, \mathrm{n}=40,268$

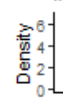
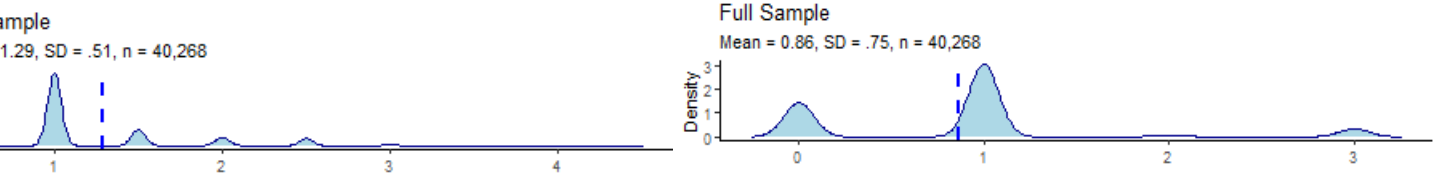

Full Sample

Mean $=0.86, \mathrm{SD}=.75, \mathrm{n}=40,268$ 
Figure 2 Cont. Ridge (density) plots for each health behaviour across clusters and final sample
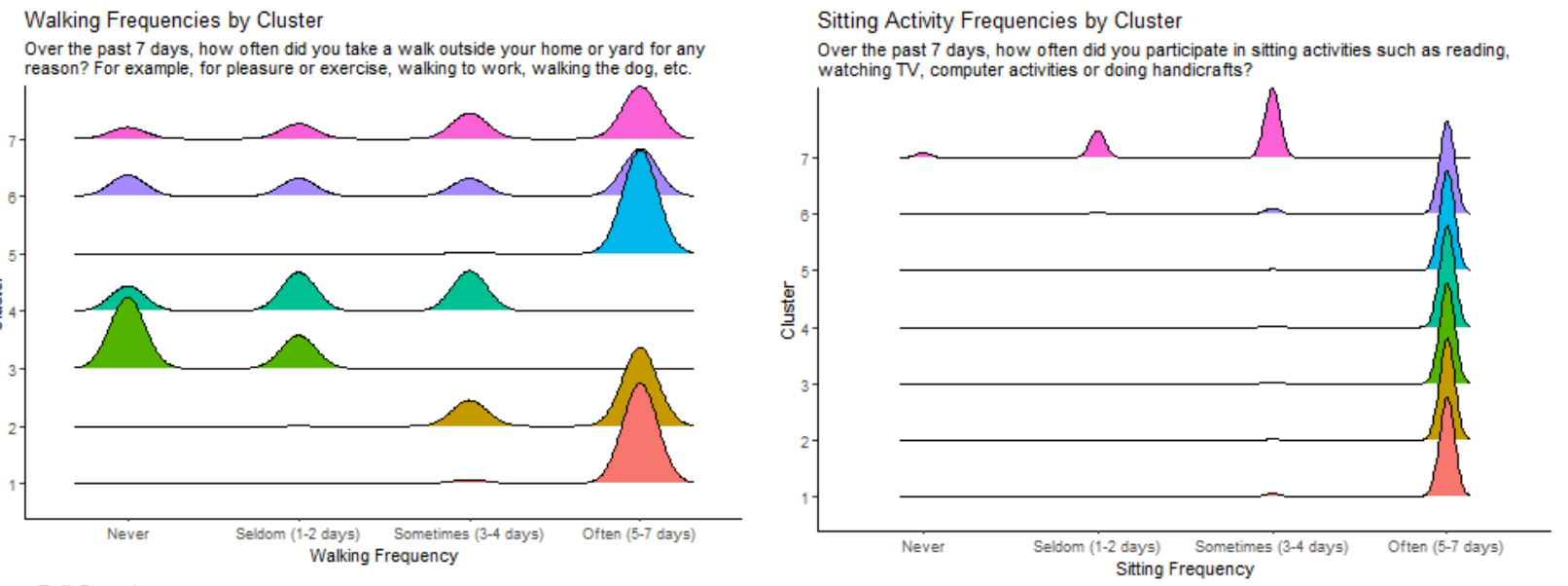

\section{Full Sample}

Mean $=3.05, \mathrm{SD}=1.13, \mathrm{n}=40,268$
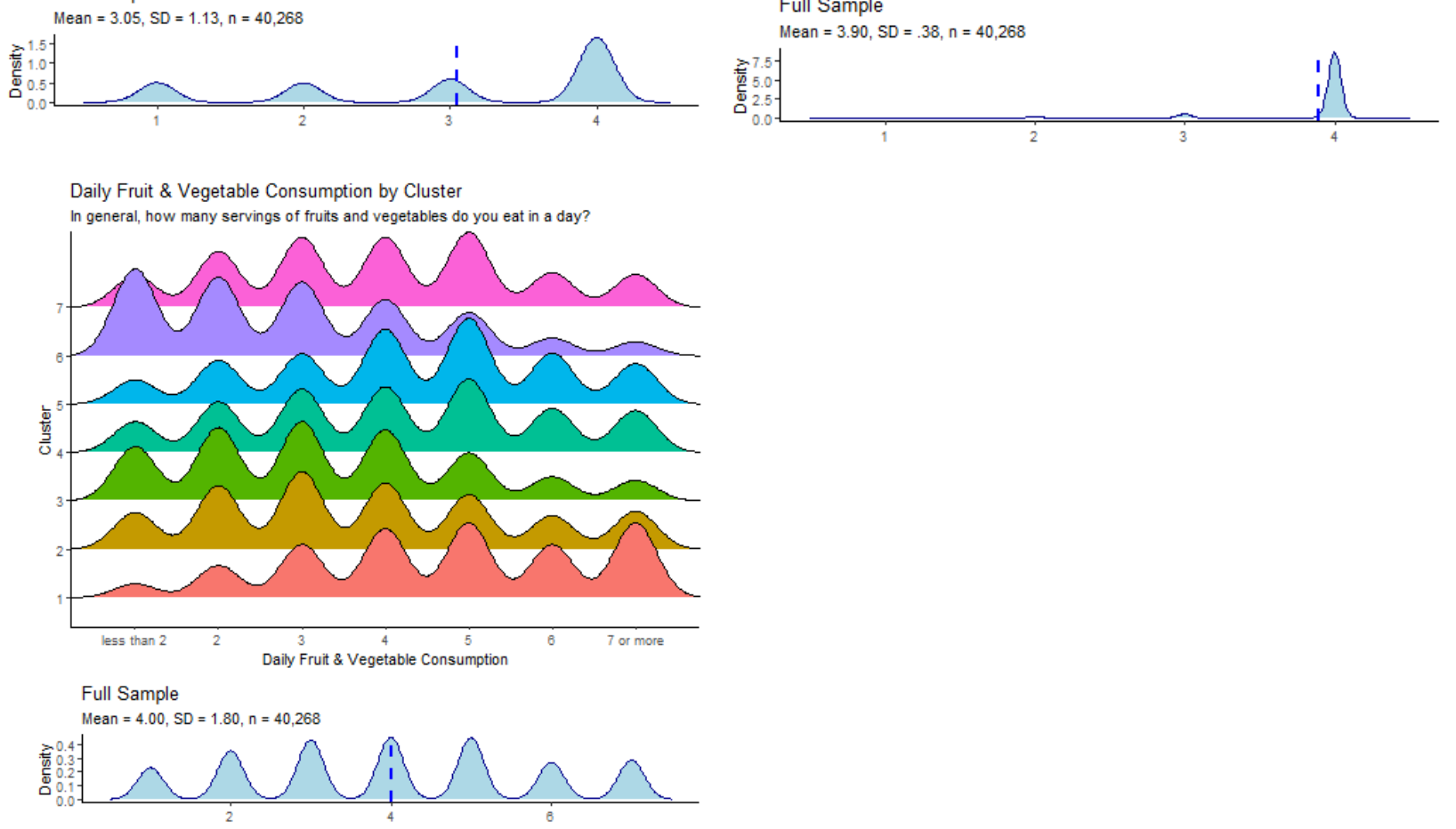

Figure note: Cluster labels are as follows: Cluster 1 = Physically Active Healthy Eaters; Cluster $2=$ Frequent Walkers with Infrequent Strenuous Exercise and Infrequent Alcohol Use; Cluster 3 = Infrequent Alcohol Use, Walking, Light Sports, and Exercise; Cluster $4=$ Frequent Alcohol Use and Infrequent Walkers; Cluster 5 = Frequent Walkers with Infrequent Strenuous Exercise but Higher Alcohol Use; Cluster $6=$ Occasional and Daily Smokers who Infrequently Eat Fruits and Vegetables and Exercise; Cluster 7 = Infrequent Sedentary Activities. 
Table 2. Counts and percentages of sociodemographic variables for final sample and each cluster

\begin{tabular}{|c|c|c|c|c|c|c|c|c|c|c|c|c|c|c|c|c|c|c|c|c|c|c|c|}
\hline & \multicolumn{2}{|c|}{ Full Sample } & \multicolumn{3}{|c|}{ Cluster 1} & \multicolumn{3}{|c|}{ Cluster 2} & \multicolumn{3}{|c|}{ Cluster 3} & \multicolumn{3}{|c|}{ Cluster 4} & \multicolumn{3}{|c|}{ Cluster 5} & \multicolumn{3}{|c|}{ Cluster 6} & \multicolumn{3}{|c|}{ Cluster 7} \\
\hline & $\mathrm{N}$ & $\%$ & $\mathrm{~N}$ & $\%$ & $\%=\%$ & $\mathrm{~N}$ & $\%$ & $\%=\%$ & $\mathrm{~N}$ & $\%$ & $\%=\%$ & $\mathrm{~N}$ & $\%$ & $\%=\%$ & $\mathrm{~N}$ & $\%$ & $\%=\%$ & $\mathrm{~N}$ & $\%$ & $\%=\%$ & $\mathrm{~N}$ & $\%$ & $\%=\%$ \\
\hline \multicolumn{24}{|l|}{ Age Group } \\
\hline $45-54$ & 10923 & 27.1 & 2274 & 29.5 & 2.4 & 1852 & 25.6 & -1.5 & 1013 & 24.7 & -2.4 & 2760 & 25.7 & -1.4 & 1040 & 20.0 & -7.2 & 1147 & 37.3 & 10.1 & 837 & 37.5 & 10.3 \\
\hline $55-64$ & 13186 & 32.7 & 2613 & 33.9 & 1.2 & 2300 & 31.8 & -0.9 & 1237 & 30.2 & -2.5 & 3451 & 32.2 & -0.6 & 1731 & 33.2 & 0.5 & 1182 & 38.4 & 5.6 & 672 & 30.1 & -2.7 \\
\hline $65-74$ & 9461 & 23.5 & 1772 & 23.0 & -0.5 & 1733 & 24.0 & 0.5 & 979 & 23.9 & 0.4 & 2591 & 24.2 & 0.7 & 1434 & 27.5 & 4.0 & 527 & 17.1 & -6.4 & 425 & 19.0 & -4.5 \\
\hline $75-85$ & 6698 & 16.6 & 1044 & 13.6 & -3.1 & 1338 & 18.5 & 1.9 & 865 & 21.1 & 4.5 & 1921 & 17.9 & 1.3 & 1007 & 19.3 & 2.7 & 223 & 7.2 & -9.4 & 300 & 13.4 & -3.2 \\
\hline NA & 0 & 0.0 & 0 & 0.0 & 0.0 & 0 & 0.0 & 0.0 & 0 & 0.0 & 0.0 & 0 & 0.0 & 0.0 & 0 & 0.0 & 0.0 & 0 & 0.0 & 0.0 & 0 & 0.0 & 0.0 \\
\hline \multicolumn{24}{|c|}{ Employment Status } \\
\hline Yes & 16401 & 40.7 & 3375 & 43.8 & 3.1 & 2843 & 39.4 & -1.4 & 1524 & 37.2 & -3.5 & 4310 & 40.2 & -0.5 & 1794 & 34.4 & -6.3 & 1411 & 45.8 & 5.1 & 1144 & 51.2 & 10.5 \\
\hline No & 1609 & 4.0 & 236 & 3.1 & -0.9 & 325 & 4.5 & 0.5 & 204 & 5.0 & 1.0 & 327 & 3.0 & -0.9 & 158 & 3.0 & -1.0 & 267 & 8.7 & 4.7 & 92 & 4.1 & 0.1 \\
\hline NA & 22258 & 55.3 & 4092 & 53.1 & -2.2 & 4055 & 56.1 & 0.9 & 2366 & 57.8 & 2.5 & 6086 & 56.8 & 1.5 & 3260 & 62.5 & 7.3 & 1401 & 45.5 & -9.8 & 998 & 44.7 & -10.6 \\
\hline \multicolumn{24}{|l|}{ Sex } \\
\hline $\mathrm{F}$ & 20220 & 50.2 & 3729 & 48.4 & -1.8 & 4029 & 55.8 & 5.6 & 2409 & 58.8 & 8.6 & 5025 & 46.9 & -3.4 & 2310 & 44.3 & -5.9 & 1563 & 50.8 & 0.6 & 1155 & 51.7 & 1.5 \\
\hline$M$ & 20048 & 49.8 & 3974 & 51.6 & 1.8 & 3194 & 44.2 & -5.6 & 1685 & 41.2 & -8.6 & 5698 & 53.1 & 3.4 & 2902 & 55.7 & 5.9 & 1516 & 49.2 & -0.5 & 1079 & 48.3 & -1.5 \\
\hline NA & 0 & 0.0 & 0 & 0.0 & 0.0 & 0 & 0.0 & 0.0 & 0 & 0.0 & 0.0 & 0 & 0.0 & 0.0 & 0 & 0.0 & 0.0 & 0 & 0.0 & 0.0 & 0 & 0.0 & 0.0 \\
\hline \multicolumn{24}{|c|}{ Annual Income } \\
\hline$<\$ 20 k$ & 1669 & 4.1 & 208 & 2.7 & -1.4 & 409 & 5.7 & 1.5 & 280 & 6.8 & 2.7 & 229 & 2.1 & -2.0 & 122 & 2.3 & -1.8 & 326 & 10.6 & 6.4 & 95 & 4.3 & 0.1 \\
\hline$\$ 20-\$ 49 k$ & 8663 & 21.5 & 1360 & 17.7 & -3.9 & 1912 & 26.5 & 5.0 & 1144 & 27.9 & 6.4 & 1973 & 18.4 & -3.1 & 931 & 17.9 & -3.7 & 865 & 28.1 & 6.6 & 478 & 21.4 & -0.1 \\
\hline$\$ 50-\$ 99 k$ & 13928 & 34.6 & 2526 & 32.8 & -1.8 & 2464 & 34.1 & -0.5 & 1407 & 34.4 & -0.2 & 3823 & 35.7 & 1.1 & 1941 & 37.2 & 2.7 & 999 & 32.4 & -2.1 & 768 & 34.4 & -0.2 \\
\hline$\$ 100-\$ 149 k$ & 7439 & 18.5 & 1636 & 21.2 & 2.8 & 1117 & 15.5 & -3.0 & 600 & 14.7 & -3.8 & 2195 & 20.5 & 2.0 & 1038 & 19.9 & 1.4 & 440 & 14.3 & -4.2 & 413 & 18.5 & 0.0 \\
\hline$\$ 150 \mathrm{k}+$ & 6214 & 15.4 & 1578 & 20.5 & 5.1 & 841 & 11.6 & -3.8 & 375 & 9.2 & -6.3 & 1886 & 17.6 & 2.2 & 912 & 17.5 & 2.1 & 260 & 8.4 & -7.0 & 362 & 16.2 & 0.8 \\
\hline NA & 2355 & 5.8 & 395 & 5.1 & -0.7 & 480 & 6.6 & 0.8 & 288 & 7.0 & 1.2 & 617 & 5.8 & -0.1 & 268 & 5.1 & -0.7 & 189 & 6.1 & 0.3 & 118 & 5.3 & -0.6 \\
\hline \multicolumn{24}{|c|}{$\mathrm{M}$ arital Status } \\
\hline Single & 3230 & 8.0 & 580 & 7.5 & -0.5 & 685 & 9.5 & 1.5 & 355 & 8.7 & 0.7 & 681 & 6.4 & -1.7 & 292 & 5.6 & -2.4 & 465 & 15.1 & 7.1 & 172 & 7.7 & -0.3 \\
\hline Married/CL & 28520 & 70.8 & 5692 & 73.9 & 3.1 & 4843 & 67.0 & -3.8 & 2673 & 65.3 & -5.5 & 8076 & 75.3 & 4.5 & 3972 & 76.2 & 5.4 & 1697 & 55.1 & -15.7 & 1567 & 70.1 & -0.7 \\
\hline Widowed & 3600 & 8.9 & 568 & 7.4 & -1.6 & 765 & 10.6 & 1.7 & 501 & 12.2 & 3.3 & 868 & 8.1 & -0.8 & 428 & 8.2 & -0.7 & 274 & 8.9 & -0.0 & 196 & 8.8 & -0.2 \\
\hline Divorced & 3879 & 9.6 & 684 & 8.9 & -0.8 & 753 & 10.4 & 0.8 & 458 & 11.2 & 1.6 & 871 & 8.1 & -1.5 & 413 & 7.9 & -1.7 & 476 & 15.5 & 5.8 & 224 & 10.0 & 0.4 \\
\hline Separated & 1027 & 2.6 & 175 & 2.3 & -0.3 & 177 & 2.5 & -0.1 & 105 & 2.6 & 0.0 & 225 & 2.1 & -0.5 & 107 & 2.1 & -0.5 & 165 & 5.4 & 2.8 & 73 & 3.3 & 0.7 \\
\hline NA & 12 & 0.0 & 4 & 0.1 & 0.0 & 0 & 0.0 & -0.0 & 2 & 0.0 & 0.0 & 2 & 0.0 & -0.0 & 0 & 0.0 & -0.0 & 2 & 0.1 & 0.0 & 2 & 0.1 & 0.1 \\
\hline \multicolumn{24}{|c|}{ Notes: $\%-\%$ denotesthe $\%$ difference between final sample and a given cluster and are colorized to highlight the size (color intensity) and direction (green = positive, red $=$ negative) of the differences. } \\
\hline $\begin{array}{l}\text { Cluster labels ar } \\
\text { Walking, Light } \$\end{array}$ & folo & $\begin{array}{l}\text { s: } c \\
d E x e\end{array}$ & $\begin{array}{l}\text { ster } 1=\mathrm{F} \\
\text { ise; Clus }\end{array}$ & $\begin{array}{l}\text { Physica } \\
\text { ster } 4 \text { = }\end{array}$ & Ally Active & Health & $\begin{array}{l}\text { y Eaters } \\
\text { Usear }\end{array}$ & $\begin{array}{l}\text { s; Cluster } \\
\text { nd Infreq }\end{array}$ & $\begin{array}{l}2=\text { Fre } \\
\text { uent Wa }\end{array}$ & $\begin{array}{l}\text { equent } \mathrm{V} \\
\text { lalkers; } \mathrm{C}\end{array}$ & $\begin{array}{l}\text { Nalkers w } \\
\text { Cluster } 5\end{array}$ & $\begin{array}{l}\text { ith Infre } \\
=\text { Freque }\end{array}$ & $\begin{array}{l}\text { quent s } \\
\text { ent Wal }\end{array}$ & kers with & Fxerris & end & equent & Aloohol & $\begin{array}{l}\text { Use; C } \\
\text { ut Highe }\end{array}$ & $\begin{array}{l}\text { Cluster } 3= \\
\text { er Alcoho }\end{array}$ & $=$ Infrequ & $\begin{array}{l}\text { ent Alco } \\
\text { luster } 6\end{array}$ & \\
\hline
\end{tabular}


Cluster 1: Physically Active Healthy Eaters. People assigned to this cluster comprised 19\% of all participants and engaged in more walking activities and exercise than the final sample and ate slightly more daily servings of fruits and vegetables ${ }^{3}$. Specifically, people in Cluster 1 engaged in walking activities, on average, closer to 6-7 days a week than the 3-4 days overall average $(M=4.0 ; S D=0.2)$ and strenuous exercise between $1-2$ days a week and 3-4 days a week $(M=2.4$; $S D=0.7)$. Average daily fruit and vegetable consumption was closer to 5 servings per day $(M=4.7 ; S D=1.7)$ compared to the overall average of 4 servings per day. When compared to the proportion of people earning $\$ 150,000$ or more annually in the final sample $(15.4 \%)$, more people in this cluster earned $\$ 150,000$ or more $(20.5 \%)$.

Cluster 2: Frequent Walkers with Infrequent Strenuous Exercise and Alcohol Use. People in this cluster represented $18 \%$ of participants and engaged in more frequent walking activities but less frequent strenuous exercise and alcohol consumption when compared to the overall sample. Walking activities were closer to 6-7 days a week than 3-4 days $(\mathrm{M}=3.8 ; \mathrm{SD}=0.4)$ while the weekly average for strenuous exercise was closer to 'never' than 'seldom' $(M=1.2$; $\mathrm{SD}=.4$ ) and alcohol consumption was closer to monthly than daily $(\mathrm{M}=2.7 ; \mathrm{SD}=1.6)$. Demographically, there were $5.6 \%$ fewer males in this group than the final sample and $5 \%$ more people earning $\$ 20,000-\$ 49,000$ annually.

Cluster 3: Infrequent Alcohol Use, Walking, Light Sports, and Exercise. In this group (10\% of participants), all health behaviours were performed less frequently than the group average except for slightly more sitting activities. Notably, the frequencies of walking, light physically activity, and strenuous exercise were each closer to 'never' than 'seldom (1-2 days)' $(\mathrm{M}=1.3$, $1.2,1.3 ; \mathrm{SD}=0.5,0.4,0.6)$. Alcohol consumption was lower than average $(\mathrm{M}=2.3 ; \mathrm{SD}=1.5)$ indicating that people in this group consumed alcohol closer to monthly than daily. Daily fruit and vegetable servings were closer to 3 servings a week $(M=3.4 ; S D=1.7)$ than the overall average of 4 servings $(M=4.0 ; S D=1.8)$. Additionally, the group was comprised of non-smokers. Demographically, there are more people aged 78-85 in this group $(21.1 \%)$ compared to overall (16.6\%), less Males (41.2\%) than overall (49.8\%), and the distribution of annual income was skewed towards lower income brackets compared to the final sample with $6.4 \%$ more people in Cluster 3 than the final sample earning $\$ 20,000-\$ 49,000$ and $6.3 \%$ less people earning $\$ 150,000$ per year or more.

Cluster 4: Frequent Alcohol Use and Infrequent Walkers. The largest of the seven clusters (27\%) was defined by near average frequencies of health behaviours with two exceptions. First, the average frequency of walking activities was lower in this cluster with people engaging in walking activities 1-2 days per week $(\mathrm{M}=2.1 ; \mathrm{SD}=0.8)$ compared to 3-4 days per week in the final sample $(M=3.1 ; S D=1.1)$. Second, alcohol consumption was higher $(M=5.3 ; S D=$ 1.4) than the final sample average $(M=4.2 ; S D=2.0)$ meaning that people in this cluster were closer to daily alcohol consumption than monthly consumption on the 1 (< once a month) to 7 (almost every day) scale. Demographically, there were slightly more people in married or common law relationships in this cluster (75.3\%) compared to the overall sample (70.8\%).

Cluster 5: Frequent Walkers with Infrequent Strenuous Exercise but Higher Alcohol Use. Comprised of $13 \%$ of participants, Cluster 5 is similar to Cluster 2 with higher than average walking frequencies $(\mathrm{M}=4.0 ; \mathrm{SD}=0.1)$ and lower than average strenuous exercise $(\mathrm{M}=$ $1.2 ; \mathrm{SD}=0.3$ ). However, these two clusters are differentiated by alcohol consumption with the average drinking frequency for this group being 1 point away from 'almost every day' on a 17 scale $(M=6.0 ; S D=1.0)$. Differences in demographics also distinguish these two clusters. Specifically, there are fewer people aged $45-54$ in this cluster compared to overall (20.0\% vs

\footnotetext{
${ }^{3}$ We conducted one-way ANOVAs and follow-up multiple comparison tests as planned in our protocol. All oneway ANOVAs were statistically significant. Twenty-one follow-up tests using Tukey HSD were conducted per health behaviour resulting in 147 follow-up comparisons. Overall, 93.2\% of comparisons were statistically significant. (see online materials for full analysis). Given that most differences were statistically significant, we omit the reporting of p-values and interpret the clusters descriptively (although all mean level differences presented descriptively are also statistically significant).
} 
$27.1 \%$ ), more Males (55.7\% vs $49.8 \%$ ), and more people in married or common law relationships $(76.2 \%$ vs $70.8 \%)$.

Cluster 6: Occasional and Daily Smokers who Infrequently Eat Fruits and Vegetables and Exercise. Nearly all participants who smoked occasionally or daily were included in this cluster ( $8 \%$ of total). Participants in this cluster also ate, on average, 1 less serving of fruits and vegetables per week $(M=3.0 ; S D=1.7)$ compared to the overall sample $(M=4.0 ; S D=1.8)$. Additionally, the average level of strenuous exercise in this group was closer to 'never' $(\mathrm{M}=$ $1.3 ; \mathrm{SD}=0.6)$ than the overall sample whose average was closer to 'seldom (1-2 days per week'; $\mathrm{M}=1.6$; $\mathrm{SD}=0.8$ ). Demographically, this group was skewed towards younger age groups (e.g., $37.3 \%$ aged $45-54$ vs $27.1 \%$ final sample) and lower income brackets (e.g., $10.6 \%$ with income $<\$ 20,000$ vs $4.1 \%$ final sample). Lastly, this group was comprised of $15.7 \%$ less married or common law individuals, compared to overall, and $7.1 \%$ more single people and $5.8 \%$ more divorced participants.

Cluster 7: Infrequent Sedentary Activities. The smallest cluster by group membership (6\%), people assigned to this cluster engaged in sitting activities, on average, between 'seldom (1-2 days' and 'sometimes ( $2-4$ days)' $(\mathrm{M}=2.6$; $\mathrm{SD}=0.6$ ) compared to the overall sample who, on average, participated in sitting activities closer to 'often (5-7 days)' $(\mathrm{M}=3.9 ; \mathrm{SD}=0.4)$. Demographically, this group contains $10.3 \%$ more people aged $45-54$ than the overall sample.

\section{Multinomial Logistic Regressions}

We conducted four multinomial logistic regressions predicting cluster membership to determine whether clusters are associated with 1) sociodemographic factors, 2) indicators of physical and mental health, 3) non-health behaviours, and 4) health care utilization. Analysis was performed using the 'multinom' function from the 'nnet' package (Ripley et al., 2016). The reference group for the regression analysis was Cluster 4 ('frequent alcohol use and infrequent walkers') due to most health behaviours being close to the final sample average. Results are presented in Tables 3 (sociodemographics), 4 (heath indicators), and 5 (health care usage). A summary of the findings follows each table.

Sociodemographics. Several patterns of increasing or decreasing log of the odds ratios were observed for ordinal predictor variables (i.e., age and income). For age groups, the odds of being in Cluster 5 ('frequent walkers with infrequent strenuous exercise but higher alcohol use"), relative to Cluster 4 ('frequent alcohol use and infrequent walkers"), increased with older age. The odds of being assigned to Cluster 6 ('occasional and daily smokers who infrequently eat fruits and vegetables and exercise') and Cluster 7 ("infrequent sitting activities") decreased with older age, especially with the occasional and daily smokers group and those aged 75-85 $(O R=.04, C I=.01, .07, p<.001)$. For annual income, the odds of being assigned to Cluster 2 ("frequent walkers with infrequent strenuous exercise and alcohol use"), Cluster 3 ('infrequent alcohol use, walking, light sports, and exercise"), Cluster 6 ("occasional and daily smokers who infrequently eat fruits and vegetables and exercise'), and Cluster 7 ('infrequent sitting activities') each decreased with higher levels of income. The strongest association was observed between those reporting an annual income of \$150,000 or greater and membership in Cluster 6 ('occasional and daily smokers who infrequently eat fruits and vegetables and exercise'; $O R=.09, C I=.06, .13, p<0.001)$. 
Figure 3. Radar plots for each cluster (standardized means).

Cluster 1:

Physically Active Healthy Eaters
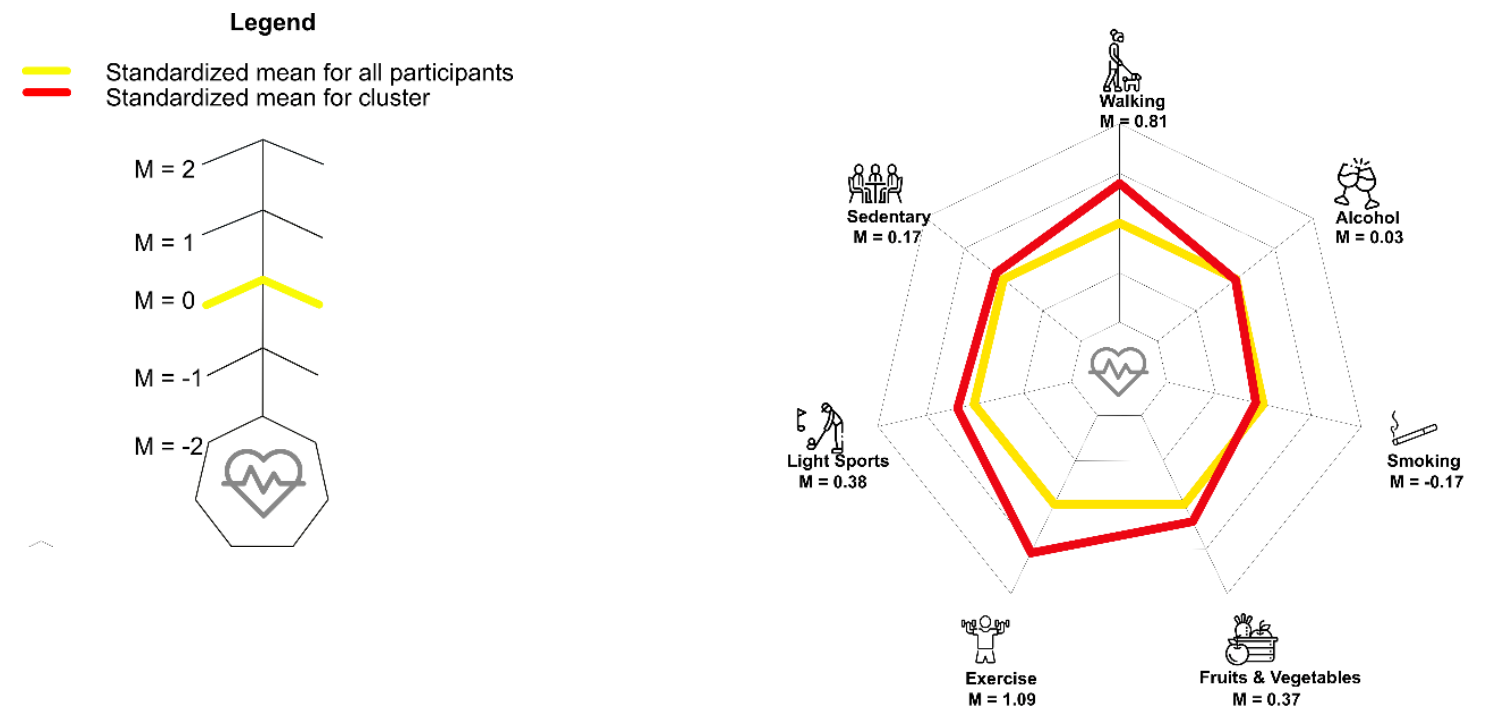

Cluster 2:

Frequent Walkers with Infrequent Strenuous Exercise and Alcohol Use
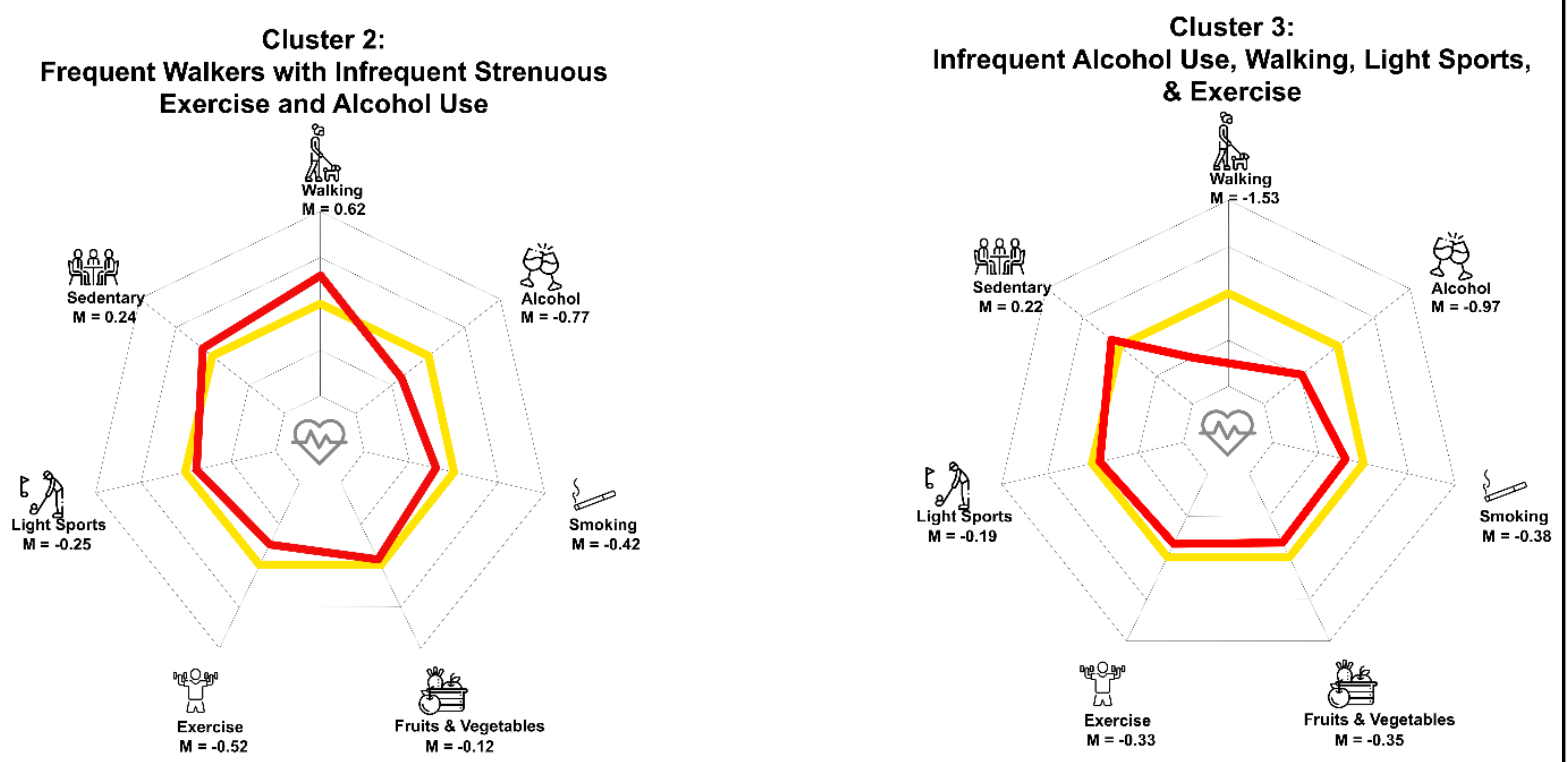
Figure 3 Continued. Radar plots for each cluster (standardized means).

Cluster 4:

Frequent Alcohol Use \& Infrequent Walkers

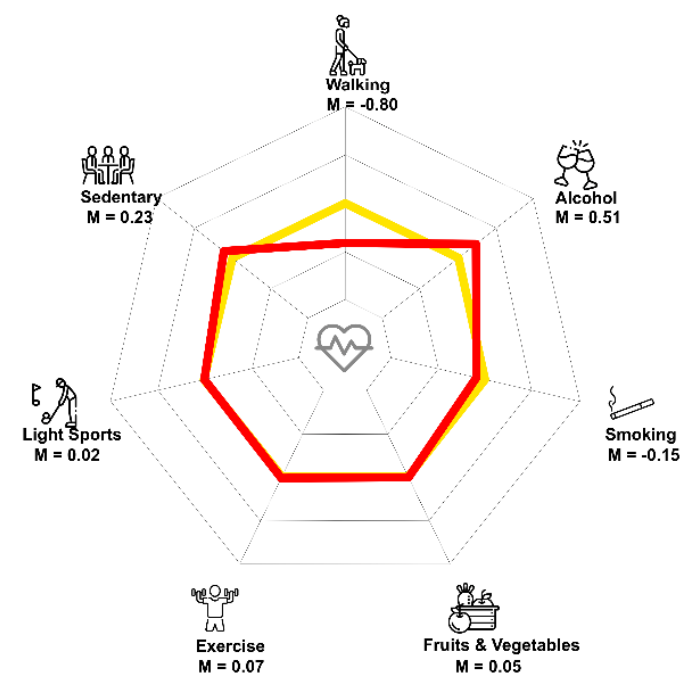

Cluster 6:

Occasional and Daily Smokers who Infrequently Eat Fruits and Vegetables and Exercise
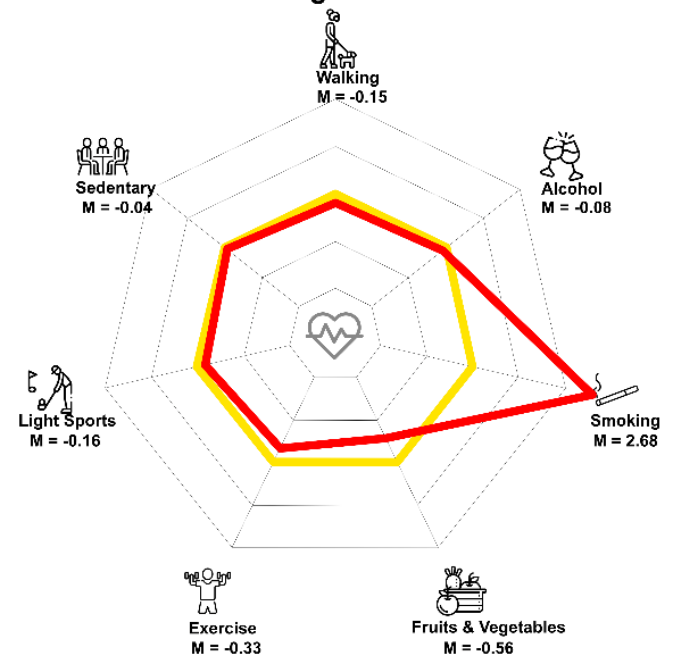

Cluster 5:

Frequent Walkers with Infrequent Strenuous Exercise but Higher Alcohol Use

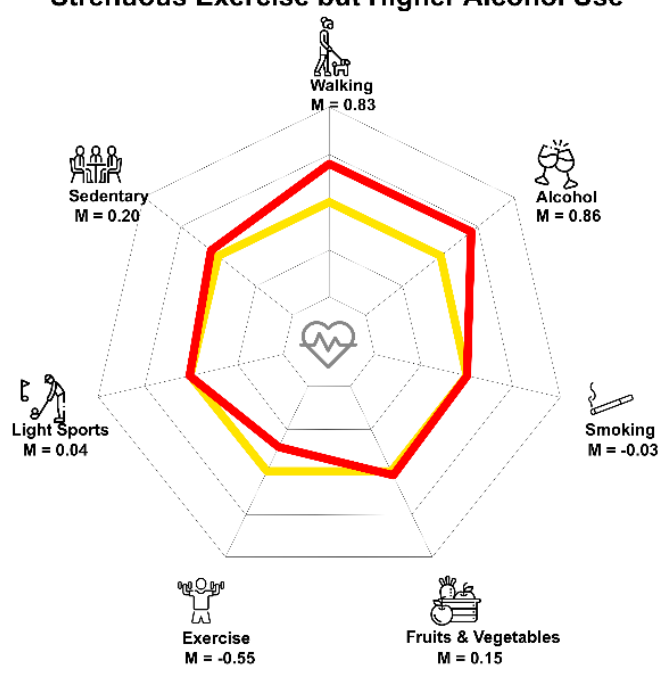

Cluster 7:

Infrequent Sedentary Activities

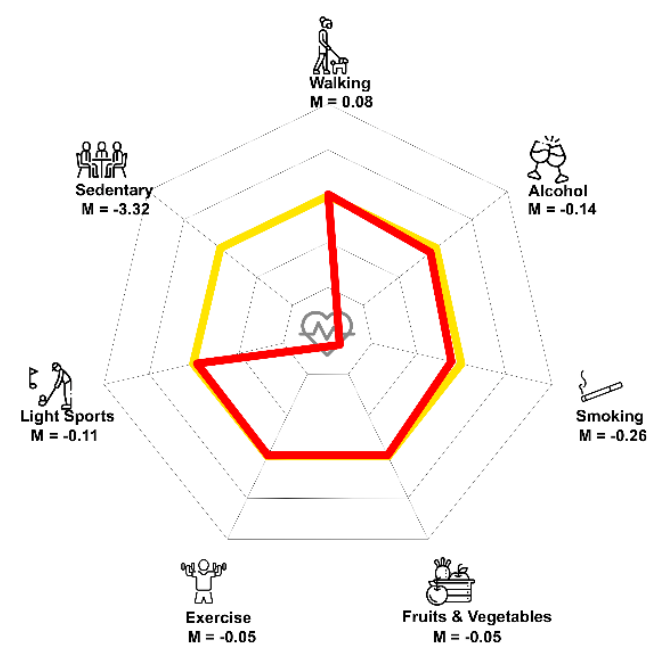


Table 3. Multinomial logistic regression with sociodemographic variables predicting cluster membership

\begin{tabular}{|c|c|c|c|c|c|c|c|c|c|c|c|c|c|c|c|c|c|c|}
\hline \multirow[b]{2}{*}{ Characteristic } & \multicolumn{3}{|c|}{ Cluster 1} & \multicolumn{3}{|c|}{ Cluster 2} & \multicolumn{3}{|c|}{ Cluster 3} & \multicolumn{3}{|c|}{ Cluster 5} & \multicolumn{3}{|c|}{ Cluster 6} & \multicolumn{3}{|c|}{ Cluster 7} \\
\hline & OR & $95 \% \mathrm{Cl}^{7}$ & p-value & OR & $95 \% \mathrm{Cl}^{1}$ & p-value & OR & $95 \% \mathrm{Cl}^{\prime}$ & $\mathrm{p}$-value & OR & $95 \% \mathrm{Cl}^{\prime}$ & p-value & OR & $95 \% \mathrm{Cl}^{\prime}$ & $\mathrm{p}$-value & OR & $95 \% \mathrm{Cl}^{7}$ & p-value \\
\hline \multicolumn{19}{|l|}{ Age_Group } \\
\hline $45-54$ & - & - & & - & - & & - & - & & - & - & & - & - & & - & - & \\
\hline $55-64$ & 0.89 & $0.81,1.0$ & 0.021 & 1.0 & $0.90,1.1$ & 0.9 & 1.0 & $0.88,1.1$ & $>0.9$ & 1.3 & $1.1,1.4$ & $<0.001$ & 0.76 & $0.67,0.87$ & $<0.001$ & 0.70 & $0.61,0.81$ & $<0.001$ \\
\hline $65-74$ & 0.77 & $0.63,0.94$ & 0.011 & 0.95 & $0.77,1.2$ & 0.6 & 0.81 & $0.62,1.0$ & 0.10 & 1.5 & $1.2,1.9$ & $<0.001$ & 0.31 & $0.22,0.43$ & $<0.001$ & 0.56 & $0.41,0.77$ & $<0.001$ \\
\hline $75-85$ & 0.70 & $0.47,1.0$ & 0.075 & 0.85 & $0.58,1.3$ & 0.4 & 0.95 & $0.61,1.5$ & 0.8 & 1.7 & $1.2,2.5$ & 0.006 & 0.04 & $0.01,0.17$ & $<0.001$ & 0.48 & $0.26,0.90$ & 0.023 \\
\hline
\end{tabular}

Sex

\begin{tabular}{|c|c|c|c|c|c|c|c|c|c|c|c|c|c|c|c|c|c|c|}
\hline Female & - & - & & - & - & & - & - & & - & - & & - & - & & - & - & \\
\hline Male & 0.86 & $0.79,0.95$ & 0.002 & 0.74 & $0.67,0.82$ & $<0.001$ & 0.75 & $0.66,0.84$ & $<0.001$ & 0.87 & $0.77,1.0$ & 0.011 & 0.90 & $0.80,1.0$ & 0.085 & 0.82 & $0.72,0.93$ & 0.003 \\
\hline
\end{tabular}

\section{M arital_Status}

\begin{tabular}{|c|c|c|c|c|c|c|c|c|c|c|c|c|c|c|c|c|c|c|}
\hline Single & - & - & & - & - & & - & - & & - & - & & - & - & & - & - & \\
\hline Married/CommonLaw & 0.90 & $0.76,1.1$ & 0.2 & 0.95 & $0.80,1.1$ & 0.5 & 1.2 & $1.0,1.5$ & 0.088 & 1.0 & $0.84,1.3$ & 0.7 & 0.71 & $0.58,0.86$ & $<0.001$ & 1.0 & $0.76,1.2$ & 0.8 \\
\hline Widowed & 1.2 & $0.83,1.7$ & 0.4 & 1.0 & $0.72,1.4$ & $>0.9$ & 1.0 & $0.69,1.6$ & 0.8 & 1.1 & $0.74,1.7$ & 0.6 & 1.0 & $0.67,1.5$ & $>0.9$ & 1.2 & $0.76,2.0$ & 0.4 \\
\hline Divorced & 1.0 & $0.82,1.3$ & 0.8 & 0.87 & $0.70,1.1$ & 0.2 & 1.1 & $0.85,1.4$ & 0.5 & 1.0 & $0.79,1.4$ & 0.7 & 1.0 & $0.81,1.3$ & 0.9 & 1.0 & $0.71,1.3$ & 0.8 \\
\hline Separated & 1.1 & $0.78,1.4$ & 0.7 & 1.0 & $0.70,1.3$ & 0.8 & 1.0 & $0.67,1.4$ & $>0.9$ & 1.1 & $0.73,1.6$ & 0.7 & 1.1 & $0.81,1.5$ & 0.5 & 1.4 & $0.95,2.1$ & 0.091 \\
\hline
\end{tabular}

\begin{tabular}{|c|c|c|c|c|c|c|c|c|c|c|c|c|c|c|c|c|c|c|}
\hline Income & & & & & & & & & & & & & & & & & & \\
\hline$\$ 20 \mathrm{k}$ & - & - & & - & - & & - & - & & - & - & & - & - & & - & - & \\
\hline$\$ 20-\$ 49 k$ & 0.67 & $0.44,1.0$ & 0.056 & 0.46 & $0.32,0.66$ & $<0.001$ & 0.49 & $0.33,0.73$ & $<0.001$ & 0.68 & $0.41,1.1$ & 0.13 & 0.48 & $0.33,0.70$ & $<0.001$ & 0.60 & $0.36,1.0$ & 0.048 \\
\hline$\$ 50-\$ 99 k$ & 0.62 & $0.41,0.92$ & 0.019 & 0.35 & $0.25,0.50$ & $<0.001$ & 0.31 & $0.21,0.47$ & $<0.001$ & 0.75 & $0.46,1.2$ & 0.2 & 0.26 & $0.18,0.37$ & $<0.001$ & 0.50 & $0.31,0.82$ & 0.006 \\
\hline$\$ 100-\$ 149 k$ & 0.64 & $0.43,1.0$ & 0.031 & 0.27 & $0.19,0.38$ & $<0.001$ & 0.23 & $0.15,0.34$ & $<0.001$ & 0.77 & $0.47,1.3$ & 0.3 & 0.15 & $0.10,0.21$ & $<0.001$ & 0.40 & $0.24,0.66$ & $<0.001$ \\
\hline$\$ 150 \mathrm{k}+$ & 0.75 & $0.50,1.1$ & 0.2 & 0.21 & $0.15,0.31$ & $<0.001$ & 0.15 & $0.10,0.23$ & $<0.001$ & 0.87 & $0.53,1.4$ & 0.6 & 0.09 & $0.06,0.13$ & $<0.001$ & 0.36 & $0.22,0.60$ & $<0.001$ \\
\hline Social_Support & 1.0 & $1.0,1.0$ & $>0.9$ & 1.0 & $1.0,1.0$ & 0.6 & 1.0 & $1.0,1.0$ & $<0.001$ & 1.0 & $1.0,1.0$ & 0.9 & 1.0 & $1.0,1.0$ & 0.5 & 1.0 & $1.0,1.0$ & 0.8 \\
\hline \multicolumn{19}{|l|}{ Employed } \\
\hline Yes & - & - & & - & - & & - & - & & - & - & & - & - & & - & - & \\
\hline No & 0.89 & $0.74,1.1$ & 0.2 & 1.1 & $0.94,1.3$ & 0.2 & 1.3 & $1.0,1.5$ & 0.026 & 1.1 & $0.92,1.4$ & 0.2 & 1.6 & $1.3,2.0$ & $<0.001$ & 0.88 & $0.68,1.1$ & 0.3 \\
\hline
\end{tabular}

Notes: Cluster 1 = Physically Active Healthy Eaters; Cluster 2 = Frequent Walkers with Infrequent Strenuous Exercise and Infrequent Acchol Use; Cluster $3=$ Infrequent Alcohol Use, Walking. Light Sports, and Exercise; Cluster 4 = Frequent Alcohol Use and Infrequent Walkers; Cluster $5=$ Frequent Walkers with Infrequent Strenuous Exercise but Higher Acohol Use; Cluster 6 = Occasional and Daily Smokers who Infrequenty Eat Frits and Vegetablesand Exercise; Cluster 7 = Infrequent Sedentary Activities 
With the exception of Cluster 6 ("occasional and daily smokers who infrequently eat fruits and vegetables and exercise), the odds of being male were lower than the odds of being male in the reference group (Cluster 4 ; range $=.74, .90$ ). The odds of a person in a married or common law relationship being assigned to the 'occasional and daily smokers' group was lower than being assigned to the reference group $(O R=.71, C I=.58, .56, p<0.001)$, however the remaining associations between relationship status and cluster memberships were not statistically significant or were associated with small log of the odds ratios. Similarly, social support was generally unrelated to cluster membership. Finally, for participants who are not employed the odds of being in Cluster 3 ("infrequent alcohol use, walking, light sports, and exercise"; OR $=1.3, C I=1.0,1.5, p=0.026$ ) and Cluster 6 ('occasional and daily smokers who infrequently eat fruits and vegetables and exercise'; $O R=1.6, C I=1.3,2.0, p<.001$ ) were greater than for being in the reference group.

Health Indicators. For a one-unit increase in general health, scored on a scale from 0 to 4 (where higher scores indicated higher reported general health), the odds of being assigned to Cluster 1 ("physically active health eaters") is greater (OR=1.20, $C I=1.2,1.3, p<.001)$ than being assigned to the reference group. In contrast, for each one-unit increase in general health, the odds of membership in Cluster 3 ('infrequent alcohol use, walking, light sports, and exercise'; OR = .79, $\mathrm{CI}=.75, .83, \mathrm{p}<.0001$ ) and Cluster 6 ('occasional and daily smokers who infrequently eat fruits and vegetables and exercise; $O R=.79, C I=.74, .84, p<0.001$ ) were lower relative to the reference group. For every one-unit increase in self-reported healthy aging, scored on a scale from 1-5, the odds of being assigned to Cluster 6 ('occasional and daily smokers who infrequently eat fruits and vegetables and exercise; $O R=.75, C I=.71, .80, p<0.001$ ) were lesser than being assigned to the reference group. Associations between mental health and cluster membership, and between life satisfaction and cluster membership, were generally negligible. Finally, for every one-unit increase in body mass index (range $=12.18,70.46$ ) the odds of cluster membership increase slightly (all $p s<.001$ ) for Cluster 2 ('frequent walkers with infrequent strenuous exercise and alcohol use') and Cluster 3 ('infrequent alcohol use, walking, light sports, and exercise'), and decrease slightly for Clusters 1, 5, 6, and 7 ('"physically active health eaters", "frequent walkers with infrequent strenuous exercise but higher alcohol use', 'occasional and daily smokers who infrequently eat fruits and vegetables and exercise', and "infrequent sitting activities", respectively).

Healthcare Use. Participants who had not visited an emergency department in the previous 12 months had higher odds of being assigned to Cluster 1 ('physically active healthy eaters'; $O R-1,2, C I=1.1,1.3, p<0.001$ ) and lower odds of being assigned to Cluster 3 ('infrequent alcohol use, walking, light sports, and exercise; $O R=.77, C I=.70, .85, p<.001$ ) or Cluster 6 ('occasional and daily smokers who infrequently eat fruits and vegetables and exercise'; $O R=$ $.75, C I=.68, .83, p<0.001$ ). People who had not had an overnight stay in a hospital in the past 12 months had lower odds of being in Cluster 3 ('infrequent alcohol use, walking, light sports, and exercise'; $O R=.80, C I=0.70, .91, p<0.001)$. No statistically significant associations between nursing home usage and cluster membership. 
Table 4. Multinomial logistic regression with health-indicator variables predicting cluster membership

\begin{tabular}{|c|c|c|c|c|c|c|c|c|c|c|c|c|c|c|c|c|c|c|}
\hline \multirow[b]{2}{*}{ Characteristic } & \multicolumn{3}{|c|}{ Cluster 1} & \multicolumn{3}{|c|}{ Cluster 2} & \multicolumn{3}{|c|}{ Cluster 3} & \multicolumn{3}{|c|}{ Cluster 5} & \multicolumn{3}{|c|}{ Cluster 6} & \multicolumn{3}{|c|}{ Cluster 7} \\
\hline & OR & $95 \% \mathrm{Cl}^{\dagger}$ & p-value & OR & $95 \% \mathrm{Cl}^{\prime}$ & p-value & OR & $95 \% \mathrm{Cl}^{\prime}$ & p-value & OR & $95 \% \mathrm{Cl}^{\top}$ & p-value & OR & $95 \% \mathrm{Cl}^{\prime}$ & p-value & OR & $95 \% \mathrm{Cl}^{\prime}$ & p-value \\
\hline General_Health & 1.2 & $1.2,1.3$ & $<0.001$ & 0.93 & $0.89,1.0$ & 0.003 & 0.79 & $0.75,0.83$ & $<0.001$ & 1.1 & $1.0,1.1$ & 0.030 & 0.79 & $0.74,0.84$ & $<0.001$ & 1.0 & $1.0,1.1$ & 0.2 \\
\hline M ental_Health & 0.94 & $0.90,1.0$ & 0.007 & 0.95 & $0.91,1.0$ & 0.016 & 1.0 & $1.0,1.1$ & 0.3 & 1.0 & $0.91,1.0$ & 0.082 & 1.0 & $0.95,1.1$ & $>0.9$ & 0.87 & $0.81,0.93$ & $<0.001$ \\
\hline Healthy_Aging & 1.1 & $1.1,1.2$ & $<0.001$ & 1.0 & $0.95,1.0$ & 0.8 & 0.92 & $0.87,1.0$ & 0.005 & 1.0 & $1.0,1.1$ & 0.5 & 0.75 & $0.71,0.80$ & $<0.001$ & 1.0 & $1.0,1.1$ & 0.2 \\
\hline Life_Satisfaction & 1.0 & $1.0,1.0$ & 0.028 & 1.0 & $1.0,1.0$ & 0.002 & 1.0 & $1.0,1.0$ & $<0.001$ & 1.0 & $1.0,1.0$ & $<0.001$ & 1.0 & $1.0,1.0$ & $<0.001$ & 1.0 & $1.0,1.0$ & 0.4 \\
\hline BMI & 1.0 & $1.0,1.0$ & $<0.001$ & 1.0 & $1.0,1.0$ & $<0.001$ & 1.0 & $1.0,1.1$ & $<0.001$ & 1.0 & $1.0,1.0$ & $<0.001$ & 1.0 & $0.94,1.0$ & $<0.001$ & 1.0 & $1.0,1.0$ & $<0.001$ \\
\hline
\end{tabular}

${ }^{\prime} \mathrm{Cl}=$ Confidence Interval

Notes: Cluster 1 = Physically Active Healthy Eaters; Cluster 2 = Frequent Walkers with Infrequent Strenuous Exercise and Infrequent Alcohol Use; Cluster $3=$ Infrequent Alcohol Use, Walking, Light Sports, and Exercise: Cluster 4 = Frequent Alcohol Use and Infrequent Walkers; Cluster $5=$ Frequent Walkers with Infrequent Strenuous Exercise but Higher Alcohol Use; Cluster $6=$ Oocasional and Daily Smokers who Infrequently Eat Fruits and Vegetablesand Exercise; Cluster $7=$ Infrequent Sedentary Activities

Table 5. Multinomial logistic regression with healthcare-use variables predicting cluster membership

\begin{tabular}{|c|c|c|c|c|c|c|c|c|c|c|c|c|c|c|c|c|c|c|}
\hline \multirow[b]{2}{*}{ Characteristic } & \multicolumn{3}{|c|}{ Cluster 1} & \multicolumn{3}{|c|}{ Cluster 2} & \multicolumn{3}{|c|}{ Cluster 3} & \multicolumn{3}{|c|}{ Cluster 5} & \multicolumn{3}{|c|}{ Cluster 6} & \multicolumn{3}{|c|}{ Cluster 7} \\
\hline & OR & $95 \% \mathrm{Cl}^{1}$ & p-value & OR & $95 \% \mathrm{Cl}^{\prime}$ & $\mathrm{p}$-value & OR & $95 \% \mathrm{Cl}^{\dagger}$ & p-value & OR & $95 \% \mathrm{Cl}^{+}$ & p-value & OR & $95 \% \mathrm{Cl}^{\top}$ & p-value & OR & $95 \% \mathrm{Cl}^{\dagger}$ & p-value \\
\hline \multicolumn{19}{|l|}{ Emergency_Dept } \\
\hline Yes & - & - & & - & - & & - & - & & - & - & & - & - & & - & - & \\
\hline No & 1.2 & $1.1,1.3$ & $<0.001$ & 1.0 & $0.88,1.0$ & 0.3 & 0.77 & $0.70,0.85$ & $<0.001$ & 1.0 & $1.0,1.1$ & 0.4 & 0.75 & $0.68,0.83$ & $<0.001$ & 1.0 & $0.85,1.1$ & 0.5 \\
\hline \multicolumn{19}{|l|}{ Hospital_Overnight } \\
\hline Yes & - & - & & - & - & & - & - & & - & - & & - & - & & - & - & \\
\hline No & 1.1 & $1.0,1.3$ & 0.10 & 1.0 & $0.85,1.1$ & 0.5 & 0.80 & $0.70,0.91$ & $<0.001$ & 1.0 & $0.85,1.1$ & 0.7 & 1.0 & $0.84,1.1$ & 0.8 & 1.1 & $0.91,1.3$ & 0.3 \\
\hline \multicolumn{19}{|l|}{ Nursing_Home } \\
\hline Yes & - & - & & - & - & & - & - & & - & - & & - & - & & - & - & \\
\hline No & 1.5 & $0.92,2.5$ & 0.10 & 1.3 & $0.82,2.0$ & 0.3 & 0.80 & $0.52,1.2$ & 0.3 & 1.9 & $1.1,3.5$ & 0.027 & 1.1 & $0.62,1.9$ & 0.8 & 1.2 & $0.61,2.5$ & 0.6 \\
\hline \multicolumn{19}{|c|}{ ' $\mathrm{Cl}=$ Confidence Interval } \\
\hline \multicolumn{19}{|c|}{$\begin{array}{l}\text { Notes: Cluster } 1 \text { = Physically Active Healthy Eaters; Cluster } 2=\text { Frequent Walkers with Infrequent Strenuous Exercise and Infrequent Alcohol Use; Cluster } 3=\text { Infrequent Alcohol Use, Walking, Light Sports, and Exercise; } \\
\text { Cluster } 4=\text { = Frequent Alcohol Use and Infrequent Walkers; Cluster } 5=\text { Frequent Walkers with Infrequent Strenuous Exercise but Higher Alcohol Use; Cluster } 6=\text { Oocasional and Daily Smokers who Infrequently Eat Fruits } \\
\text { and Vegetablesand Exercise; Cluster } 7=\text { Infrequent Sedentary Activities }\end{array}$} \\
\hline
\end{tabular}




\section{DISCUSSION}

Using representative data from the Canadian Longitudinal Study of Aging (CLSA)our cluster analysis produced seven groups based on similarities of frequencies in engaging in key health behaviours. Overall, clusters were differentiated by six of the seven health behaviours included in the analysis with the most variability observed in weekly walking frequency, strenuous exercise, and alcohol consumption. Specifically, three clusters were partly characterized by walking frequency and two were characterized by strenuous exercise and alcohol consumption, respectively. Of the remaining health behaviours, there was little variability in weekly 'light sports' frequencies within the seven clusters, while one cluster was generally defined by a relative extreme of a single behaviour (infrequent sedentary activities). Sociodemographic characteristics varied across several clusters while associations between self-reported physi$\mathrm{cal} /$ mental health and cluster memberships were generally small.

Direct comparisons between health behaviour clustering results between studies can be challenging due to variations in sample and measurement characteristics. However, clusters defined by engaging in health behaviours and clusters defined by smoking behaviour were present in our analysis and in comparable studies. For example, 'physically active healthy eaters' in our analysis represented $19 \%$ of the final sample while $9.3 \%$ were assigned to 'healthy lifestyle' cluster in Conry et al's (2011) analysis of Irish adults over 18 years, 25\% of people were defined by a lack of unhealthy behaviours in Schneider et al's (2009) analysis of a sample of German adults 50 years and older, and 24\% of people were defined as 'physically active, non-drinkers, who do not smoke' in a sample of American's aged 51 years and older analyzed by Shaw \& Agahi (2012). In the present study, 'physically active healthy eaters' tended to be younger in age with more people earning higher incomes than the overall sample. The association between this cluster and incomes may not be surprising as lower incomes are well established barriers to physical activity (Hawes et al., 2019) and healthy eating (Raine, 2005) which includes fruit and vegetable consumption.

In addition to a cluster defined by healthy behaviours, we also identified an 'occasional and daily smokers who infrequently eat fruits and vegetables and exercise' cluster capturing 8\% of the CLSA sample which is slightly lower than the $13 \%$ of people defined by being 'smokers with other risk behaviours in a German sample of adults aged 50 to 70 years old (Schneider et al., 2009); however, such differences might be explained by differences between age ranges and the data collection time points between studies. In the present study, people who were older, had higher annual incomes, or were in married or common law relationships were less likely to be assigned to the 'occasional and daily smokers who infrequently eat fruits and vegetables and exercise' cluster relative to the reference group. These findings are in line with what is known about links between smoking prevalence and income (Pichora et al., 2018) and marital status (Azagba \& Sharaf, 2011).

In contrast to the 'occasional and daily smokers who infrequently eat fruits and vegetables and exercise' and 'physically active healthy eaters' clusters, the remaining groups identified in this analysis do not have direct counterpoints in comparable analysis. To the best of our knowledge, no health behaviour clustering studies in adults have produced a grouping similar to the cluster we named 'infrequent alcohol use, walking, light sports, and exercise'. Behaviourally, this cluster was defined by little to no physical activity of any kind, non-smoking, and less frequent alcohol and fruits/vegetable consumption than average. The only behaviour that was above the final sample average were sedentary behaviours. People in this group tended to be older, have lower annual incomes, have higher BMI's, use healthcare services more frequently, not be employed, and be women. Taken together, the infrequent alcohol use, walking, light sports, and exercise' cluster may present a relatively homogenous behavioural sub-group to target for researchers and practitioners interested in conducting health behaviour interventions.

Three clusters were defined by combinations of walking, exercise, and alcohol consumption and each cluster was associated with different sociodemographic profiles. For example, people in Cluster 2 (' frequent walkers with infrequent strenuous exercise and alcohol use') tended to earn lower incomes and the cluster contained more females relative to the final sample while people in cluster 5 ('frequent walkers with infrequent strenuous exercise but higher alcohol 
use') tended to earn higher incomes and the cluster contained more males, more unemployed people, and more people in married or common law relationships relative to the final sample. That these clusters are defined by high vs low alcohol consumption and associated with both income and sex is consistent with existing evidence that men tend to consume more alcohol than women (Wilsnack et al., 2000; Holst et al., 2017) and affluence is associated with increased alcohol consumption in older adults (Brennan et al., 2010; Platt et al., 2010). People in Cluster 4 ('frequent alcohol use and infrequent walkers') also engaged in more walking activities and consumed more alcohol compared to the overall sample and demographically the cluster contained more people in married or common law relationships. Surprisingly, both Cluster 4 and 5 were partly defined by higher alcohol consumption and both contained more married individuals than the final sample which contrasts with existing evidence that marriage is associated with lower alcohol consumption (Dinescu et al., 2016; Liang \& Chikritzhs, 2012).

This research is subject to limitations worth noting when interpreting the findings. The health behaviours included in the cluster analysis were overrepresented with measures of physical activity as the scales used to capture physical activity in the CLSA (the PACE scale; Washburn et al., 1993) had several categories. While grouping people based on the types of physical activities scored with the PACE scale produced enabled us to explore variability in walking activities, the way in which physical activity frequency was measured and the nature of the categories made evaluating the health behaviours of this sample relative to behavioural guidelines difficult. Additionally, the health behaviours captured in the CLSA are not exhaustive and the inclusion of additional health behaviours (e.g., sleep hygiene, substance use, sexual risk behaviours) would influence cluster composition.

Methodologically, cluster analysis has advantages and disadvantages for characterizing health behaviours. For example, the clustering algorithm revealed associations that would have been overlooked with simple analyses such as correlations; three clusters were defined by varying combinations of walking frequency and alcohol consumption while correlations between the two variables (not reported) were negligible. However, clinically meaningful cooccurring behaviours which are known in the literature and present in the CLSA data were not present in the clusters. The combination of high physical activity and frequent sedentary behaviour, for example, is common in individuals who participate in sports (Weiler et al., 2015); this combination of behaviours was present in the CLSA data but was not identified with the cluster analysis. This distinction, and others, were not captured in the cluster analysis which illustrates the trade-offs between parsimony and nuance using hierarchical cluster analysis to describe co-occurring health behaviours.

Many of the items selected for planned analysis are self-report which have known and inherent strengths and weaknesses (Del Boca \& Noll, 2000). Additionally, we recognize that the analyses proposed are to some extent limited by their cross-sectional nature and the selection of variables included in the CLSA and selected for our analysis. Nevertheless, given the longitudinal nature of CLSA and planned future data releases, our analyses set the stage for planned future longitudinal analyses that extend the research questions to investigate changes in behaviour clusters as a function of time both between and within individuals. Lastly, direct comparisons between multiple health behaviour studies is difficult due to variations in sample, measurement characteristics, and inconsistent naming conventions. Although heterogeneous samples and measurement variability may be useful for establishing the presence of robust phenomena in the form of co-occurring behaviours, we encourage future analysis to clearly label clusters to include each prominent health behaviour based on clinically meaningful variability across clusters.

In conclusion, our findings show how the population can be segmented by the multiple health behaviours that characterise people's lives and that these segmented clusters are socially patterned and associated with different health outcomes. The scope, size and rigour of the CLSA dataset provided an unprecedented opportunity to investigate how health behaviours cluster. These clusters allow for contrasts to be made with comparable analyses in other countries and will help to inform the development of future health behaviour change interventions tailored to sub-populations and their sociodemographic profiles. Understanding which behaviours co-occur and for whom is an important first step towards developing tailored health behaviour change interventions. Future research will further develop our 
understanding of how clusters of health behaviours influence health outcomes over time using longitudinal data.

\section{Acknowledgements}

This research was made possible using the data/biospecimens collected by the Canadian Longitudinal Study on Aging (CLSA). Funding for the Canadian Longitudinal Study on Aging (CLSA) is provided by the Government of Canada through the Canadian Institutes of Health Research (CIHR) under grant reference: LSA 94473 and the Canada Foundation for Innovation, as well as the following provinces, Newfoundland, Nova Scotia, Quebec, Ontario, Manitoba, Alberta, and British Columbia. This research has been conducted using the CLSA dataset [Baseline Tracking Dataset version 3.4 and Comprehensive Dataset version 4.0.], under Application Number [19CA012]. The CLSA is led by Drs. Parminder Raina, Christina Wolfson and Susan Kirkland. Zack van Allen is supported by a CIHR Doctoral Award: Frederick Banting and Charles Best Canada Graduate Scholarship. Simon Bacon is supported by a CIHR-SPOR Mentoring Chair (SMC-151518) and a FRQS Chair (251618). Marta Marques is funded by a MarieSklodowska-Curie Fellowship (grant agreement no. 713567) at the ADAPT SFI Research Centre at Trinity College. Paquito Bernard was supported by Université du Québec à Montréal, Institut Universitaire de Santé Mentale de Montréal, and by a salary award from the Fonds de recherche du Québec Santé.

\section{Disclaimer}

The opinions expressed in this manuscript are the author's own and do not reflect the views of the Canadian Longitudinal Study on Aging.

\section{Ethics}

Secondary data analysis of CLSA data for this project was approved by the Ottawa Health Science Network Research Ethics Board (protocol ID \#20190506-01H). 


\section{REFERENCES}

Alashwal, H., El Halaby, M., Crouse, J. J., Abdalla, A., \& Moustafa, A. A. (2019). The application of unsupervised clustering methods to Alzheimer's Disease. Frontiers in Computational Neuroscience, 13. https://doi.org/10.3389/fncom.2019.00031

Azagba, S., \& Sharaf, M. (2011). Cigarette taxes and smoking participation: Evidence from recent tax increases in Canada. International Journal of Environmental Research and Public Health, 8, 15831600. https://doi.org/10.3390/ijerph8051583

Bacon, S. L., Campbell, T. S., \& Lavoie, K. L. (2020). Rethinking how to expand the evidence base for health behavior change in Cardiovascular Disease prevention. Journal of the American College of Cardiology, 75, 2619-2622. https://doi.org/10.1016/i.jacc.2020.03.055

Borsboom, D., \& Cramer, A. O. (2013). Network analysis: An integrative approach to the structure of psychopathology. Annual Review of Clinical Psychology, 9, 91-121.

Brennan, P. L., Schutte, K. K., \& Moos, R. H. (2010). Retired status and older adults' 10-year drinking trajectories. Journal of Studies on Alcohol and Drugs, 71, 165-168.

Brown, H., \& Presseau, J. (2018). Work me not into temptation: Exploring the relationship between work and healthy eating in dieters using data from the HILDA Survey. Australian Economic Review, 51, 368-381. https://doi.org/10.1111/1467-8462.12269

Buck, D., \& Frosini, F. (2012). Clustering of unhealthy behaviours over time: Implications for policy and practice. London: The Kings Fund, 24.

Butt, P., Beirness, D., Gliksman, L., Paradis, C., \& Stockwell, T. (2011). Alcohol and health in Canada: A summary of evidence and guidelines for low-risk drinking. Ottawa, ON: Canadian Centre on Substance Abuse.

Canadian Center on Substance Abuse and Addition. (2018). Canada's low risk alcohol consumption guidelines. Retrieved from: https://www.camh.ca/-/media/files/canadas-low-risk-guidelines-pdf.pdf

Charrad, M., Ghazzali, N., Boiteau, V., \& Niknafs, A. (2014). NbClust: An R package for determining the relevant number of clusters in a data set. Journal of Statistical Software, 61, 1-36. https://doi.org/10.18637/jss.v061.i06

Conry, M. C., Morgan, K., Curry, P., McGee, H., Harrington, J., Ward, M., \& Shelley, E. (2011). The clustering of health behaviours in Ireland and their relationship with mental health, self-rated health and quality of life. BMC Public Health, 11, 692. https://doi.org/10.1186/1471-2458-11-692

Del Boca, F. K., \& Noll, J. A. (2000). Truth or consequences: The validity of self-report data in health services research on addictions. Addiction, 95, 347-360.

Deliu, M., Sperrin, M., Belgrave, D., \& Custovic, A. (2016). Identification of asthma subtypes using clustering methodologies. Pulmonary Therapy, 2, 19-41. https://doi.org/10.1007/s41030-016-0017-z

deRuiter, W. K., Cairney, J., Leatherdale, S., \& Faulkner, G. (2016). The period prevalence of risk behavior co-occurrence among Canadians. Preventive Medicine, 85, 11-16. https://doi.org/10.1016/j.ypmed.2015.11.026

deRuiter, W. K., Cairney, J., Leatherdale, S. T., \& Faulkner, G. E. J. (2014). A longitudinal examination of the interrelationship of multiple health behaviors. American Journal of Preventive Medicine, 47, 283289. https://doi.org/10.1016/j.amepre.2014.04.019

Diener, Ed, Emmons, R. A., Larsen, R. J., \& Griffin, S. (1985). The satisfaction with life scale. Journal of Personality Assessment, 49, 71-75. https://doi.org/10.1207/s15327752jpa4901 13

Diener, Edward. (2006). Understanding scores on the satisfaction with life scale. Illinois: Psychology. University of Illinois at Urban Champaign.

Dinescu, D., Turkheimer, E., Beam, C. R., Horn, E. E., Duncan, G., \& Emery, R. E. (2016). Is marriage a buzzkill? A twin study of marital status and alcohol consumption. Journal of Family Psychology: JFP : Journal of the Division of Family Psychology of the American Psychological Association (Division 43), 30, 698-707. https://doi.org/10.1037/fam0000221

Dogra, S., Good, J., Gardiner, P. A., Copeland, J. L., Stickland, M. K., Rudoler, D., \& Buman, M. P. (2019). Effects of replacing sitting time with physical activity on lung function: An analysis of the Canadian Longitudinal Study on Aging. Health Rep, 30, 12-23. 
Fisher, E. B., Fitzgibbon, M. L., Glasgow, R. E., Haire-Joshu, D., Hayman, L. L., Kaplan, R. M., Nanney, M. S., \& Ockene, J. K. (2011). Behavior Matters. American Journal of Preventive Medicine, 40, e15e30. https://doi.org/10.1016/i.amepre.2010.12.031

Fung, G. (2001). A comprehensive overview of basic clustering algorithms.

Gower, J. C. (1971). A general coefficient of similarity and some of its properties. Biometrics, 27, 857-871. https://doi.org/10.2307/2528823

Hawes, A. M., Smith, G. S., McGinty, E., Bell, C., Bower, K., LaVeist, T. A., Gaskin, D. J., \& Thorpe, R. J. (2019). Disentangling race, poverty, and place in disparities in physical activity. International Journal of Environmental Research and Public Health, 16, 1193. https://doi.org/10.3390/ijerph16071193

Heeringa, S. G., \& Connor, J. H. (1995). Technical description of the Health and Retirement Survey sample design. Ann Arbor: University of Michigan.

Holst, C., Becker, U., Jørgensen, M. E., Grønbæk, M., \& Tolstrup, J. S. (2017). Alcohol drinking patterns and risk of diabetes: a cohort study of 70,551 men and women from the general Danish population. Diabetologia, 60, 1941-1950. https://doi.org/10.1007/s00125-017-4359-3

Keller, H. H., Goy, R., \& Kane, S.-L. (2005). Validity and reliability of SCREEN II (Seniors in the community: Risk evaluation for eating and nutrition, Version II). European Journal of Clinical Nutrition, 59, 11491157. https://doi.org/10.1038/sj.ejcn.1602225

Liang, W., \& Chikritzhs, T. (2012). Brief report: Marital status and alcohol consumption behaviours. Journal of Substance Use, 17, 84-90. https://doi.org/10.3109/14659891.2010.538463

Maechler, M., Rousseeuw, P., Struyf, A., Hubert, M., \& Hornik, K. (2012). Cluster: Cluster analysis basics and extensions. R Package Version, 1, 56.

Marengoni, A., Angleman, S., Melis, R., Mangialasche, F., Karp, A., Garmen, A., Meinow, B., \& Fratiglioni, L. (2011). Aging with multimorbidity: A systematic review of the literature. Ageing Research Reviews, 10, 430-439. https://doi.org/10.1016/j.arr.2011.03.003

Müllner, D. (2013). fastcluster: Fast hierarchical, agglomerative clustering routines for $\mathrm{R}$ and Python. Journal of Statistical Software, 53, 1-18. https://doi.org/10.18637/iss.v053.i09

Murphy, K. P. (2012). Machine Learning: A Probabilistic Perspective. MIT Press.

Noble, N., Paul, C., Turon, H., \& Oldmeadow, C. (2015). Which modifiable health risk behaviours are related? A systematic review of the clustering of Smoking, Nutrition, Alcohol and Physical activity ('SNAP') health risk factors. Preventive Medicine, 81, 16-41. https://doi.org/10.1016/i.ypmed.2015.07.003

Nunes, B. P., Flores, T. R., Mielke, G. I., Thumé, E., \& Facchini, L. A. (2016). Multimorbidity and mortality in older adults: A systematic review and meta-analysis. Archives of Gerontology and Geriatrics, 67, 130-138. https://doi.org/10.1016/j.archger.2016.07.008

Pichora, E., Polsky, J. Y., Catley, C., Perumal, N., Jin, J., \& Allin, S. (2018). Comparing individual and areabased income measures: Impact on analysis of inequality in smoking, obesity, and diabetes rates in Canadians 2003-2013. Canadian Journal of Public Health = Revue Canadienne de Santé Publique, 109, 410-418. https://doi.org/10.17269/s41997-018-0062-5

Platt, A., Sloan, F. A., \& Costanzo, P. (2010). Alcohol-consumption trajectories and associated characteristics among adults older than age 50. Journal of Studies on Alcohol and Drugs, 71, 169-179.

Prados-Torres, A., Calderón-Larrañaga, A., Hancco-Saavedra, J., Poblador-Plou, B., \& van den Akker, M. (2014). Multimorbidity patterns: A systematic review. Journal of Clinical Epidemiology, 67, 254-266. https://doi.org/10.1016/i.jclinepi.2013.09.021

Presseau, J., Tait, R. I., Johnston, D. W., Francis, J. J., \& Sniehotta, F. F. (2013). Goal conflict and goal facilitation as predictors of daily accelerometer-assessed physical activity. Health Psychology, 32(12), 1179. https://doi.org/10.1037/a0029430

Public Health Agency of Canada. (2016). How healthy are Canadians?: A trend analysis of the health of Canadians from a healthy living and chronic disease perspective. http://publications.gc.ca/collections/collection 2017/aspc-phac/HP40-167-2016-eng.pdf 
Public Health Agency of Canada. (2017). At-a-glance-The 2017 Canadian chronic disease indicators. Health Promotion and Chronic Disease Prevention in Canada, 37, 248-251. https://doi.org/10.24095/hpcdp.37.8.03

Raina, P. S., Wolfson, C., \& Kirkland, S. A. (2008). Canadian Longitudinal Study on Aging (CLSA) Protocol. Retrieved from CLSA website: https://clsa-elcv.ca/doc/511

Raina, P., Wolfson, C., Kirkland, S., \& Griffith, L. (2018). The Canadian Longitudinal Study on Aging (CLSA) Report on Health and Aging in Canada.

Raina, Parminder S., Wolfson, C., Kirkland, S. A., Griffith, L. E., Oremus, M., Patterson, C., Tuokko, H., Penning, M., Balion, C. M., \& Hogan, D. (2009). The Canadian longitudinal study on aging (CLSA). Canadian Journal on Aging/La Revue Canadienne Du Vieillissement, 28, 221-229.

Raine, K. D. (2005). Determinants of healthy eating in Canada: An overview and synthesis. Canadian Journal of Public Health, 8-14.

Rapkin, B. D., \& Luke, D. A. (1993). Cluster analysis in community research: Epistemology and practice. American Journal of Community Psychology, 21, 247-277. https://doi.org/10.1007/BF00941623

RC Team. (2019). R: A language and environment for statistical computing. Vienna, Austria: R Foundation for Statistical Computing.

Ripley, B., Venables, W., \& Ripley, M. B. (2016). Package 'nnet.' R Package Version, 7, 3-12.

Ross, R., Chaput, J. P., Giangregorio, L. M., Janssen, I., Saunders, T. J., Kho, M. E., ... \& Tremblay, M. S. (2020). Canadian 24-Hour Movement Guidelines for Adults aged 18-64 years and Adults aged 65 years or older: an integration of physical activity, sedentary behaviour, and sleep. Applied Physiology, Nutrition, and Metabolism, 45(10), S57-S102. https://doi.org/10.1139/apnm-2020-0467

Saint-Maurice, P. F., Troiano, R. P., Berrigan, D., Kraus, W. E., \& Matthews, C. E. (2018). Volume of light versus moderate-to-vigorous physical activity: Similar benefits for all-cause mortality? Journal of the American Heart Association, 7, e008815

Schneider, S., Huy, C., Schuessler, M., Diehl, K., \& Schwarz, S. (2009). Optimising lifestyle interventions: Identification of health behaviour patterns by cluster analysis in a German $50+$ survey. European Journal of Public Health, 19, 271-277. https://doi.org/10.1093/eurpub/ckn144

Shaw, B. A., \& Agahi, N. (2012). A prospective cohort study of health behavior profiles after age 50 and mortality risk. BMC Public Health, 12, 803. https://doi.org/10.1186/1471-2458-12-803

Sherbourne, C. D., \& Stewart, A. L. (1991). The MOS social support survey. Social Science \& Medicine, 32, 705-714.

Tremblay, M. S., Warburton, D. E. R., Janssen, I., Paterson, D. H., Latimer, A. E., Rhodes, R. E., Kho, M. E., Hicks, A., LeBlanc, A. G., Zehr, L., Murumets, K., \& Duggan, M. (2011). New Canadian physical activity guidelines. Applied Physiology, Nutrition, and Metabolism, 36, 36-46. https://doi.org/10.1139/H11-009

van Allen, Z., Bacon, S., Bernard, P., Brown, H., Desroches, S., Kastner, M., Lavoie, K., Marques, M., McCleary, N., Straus, S., Taljaard, M., Thavorn, K., Tomasone, J., \& Presseau, J. (2020). Clustering of healthy behaviours in Canadians-Protocol for a multiple behaviour analysis of data from the CLSA. PsyArXiv. https://doi.org/10.31234/osf.io/y2a7m

Washburn, R. A., Smith, K. W., Jette, A. M., \& Janney, C. A. (1993). The Physical Activity Scale for the Elderly (PASE): Development and evaluation. Journal of Clinical Epidemiology, 46, 153-162.

Weiler, R., Aggio, D., Hamer, M., Taylor, T., \& Kumar, B. (2015). Sedentary behaviour among elite professional footballers: Health and performance implications. BMJ Open Sport \& Exercise Medicine, 1, e000023. https://doi.org/10.1136/bmisem-2015-000023

Wilsnack, R. W., Vogeltanz, N. D., Wilsnack, S. C., \& Harris, T. R. (2000). Gender differences in alcohol consumption and adverse drinking consequences: Cross-cultural patterns. Addiction, 95, 251-265. https://doi.org/10.1046/j.1360-0443.2000.95225112.x

Wolfson, C., Raina, P. S., Kirkland, S. A., Pelletier, A., Uniat, J., Furlini, L., Angus, C. L., Strople, G., Keshavarz, H., \& Szala-Meneok, K. (2009). The Canadian community health survey as a potential recruitment vehicle for the Canadian longitudinal study on aging. Canadian Journal on Aging $=\mathrm{La}$ Revue Canadienne Du Vieillissement, 28, 243-249. https://doi.org/10.1017/S0714980809990031 
Xu, R., \& Wunsch, D. (2005). Survey of clustering algorithms. IEEE Transactions on Neural Networks, 16, 645-678. https://doi.org/10.1109/TNN.2005.845141 


\section{Appendix}

Table I. Variables to be included in analysis

Behaviour CLSA Variable

\section{Item Question \& Response Scale}

\section{Health Behaviours}

I. Sedentary Behav- PA2_SIT iour

2. Walking

\section{PA2_WALK}

3. Light / Moderate Physical Activity

4. Strenuous Physical Activity / Exercise

5. Fruit and Vegetable Consumption

6. Smoking

7. Alcohol Use

8. Sleep

9. Caregiving for Family Member
10. Puzzles/crosswords
GEN_BRD
II. Music
GEN_MUSC

12. Community Activities

SPA_DFRE

13. Social Media
Combined: PA2_MSPRT, PA2_LSPRT

Combined PA2_SSPRT, PA2 EXER

NUR_FRTVEG

SMK CURRCG

ALC_FREQ

SLE HOUR NB

"Over the past 7 days, how often did you participate in sitting activities such as reading, watching TV, computer activities or doing handicrafts?" (I (never) to 4 (often, 5 to 7 days))

"Over the past 7 days, how often did you take a walk outside your home or yard for any reason?" (I (never) to 4 (often, 5 to 7 days))

"Over the past 7 days, how often did you engage in moderate sports or recreational activities such as ballroom dancing, hunting, skating, golf without a cart, softball or other similar activities?" (I (never) to 4 (often, 5 to 7 days))

Over the past 7 days, how often did you engage in light sports or recreational activities such as bowling, golf with a cart, shuffleboard, badminton, fishing or other similar activities? (I (never) to 4 (often, 5 to 7 days))

"Over the past 7 days, how often did you engage in strenuous sports or recreational activities such as jogging, swimming, snowshoeing, cycling, aerobics, skiing, or other similar activities?" (I (never) to 4 (often, 5 to 7 days))

"Over the past 7 days, how often did you do any exercises specifically to increase muscle strength and endurance, such as lifting weights or push-ups, etc.?" (I (never) to 4 (often, 5 to 7 days))

"In general, how many servings of fruits and vegetables do you eat in a day?" ( $($ (seven or more) to 7 (less than two))

"At the present time, do you smoke cigarettes daily, occasionally or not at all?" (I (daily) 2 (occasionally) 0 (not at all))

"About how often during the past 12 months did you drink alcohol?" (I (almost every day) to 7 (less than once a month))

"During the past month, on average, how many hours of actual sleep did you get at night?" (continuous)

\section{Non-Health Behaviours}

This flag variable indicates whether the respondent provided assistance in the past 12 months to another person because of a health condition or limitation. Assistance provided as part of a volunteer organization or paid job is excluded. ( $I=$ did not provide any assistance, 2 = did not provide any assistance or only provided financial assistance)

"Playing board games, cards, crossword puzzles, jigsaw puzzles, or sudoku" ( I (every day); 2 (several times a week); 3 (several times a month); 4 (several times a year); 5 (once a year or less))

"Playing a musical instrument or singing in a choir." (I (every day); 2 (several times a week); 3 (several times a month); 4 (several times a year); 5 (once a year or less))

This variable categorizes respondents by the frequency of their participation in any type of community-related activity during the past 12 months. Activities include: I) church or religious activities, 2) attending concerts, plays, or visiting museums, 3) service club or fraternal organization activities, 4) community or professional association activities, 5) volunteer or charity work, 6) participation in family/ friends activities out of household, 7) participation in sports or physical activities with others, 8) participation in educational or cultural activities, and 9) participation in other recreational activities. Scoring: $0=$ no activities, $I=$ yearly, $2=$ monthly, $3=$ weekly, 4 = daily.

"Do you currently use social networking sites on the Internet? Examples of such sites include Facebook, Linkedln, MySpace, MSNGroups, or Twitter." (I (yes), 2 (no); if yes, code use highest frequency of indicators) 
14. Age

15. Sex

16. Country of birth

17. Marital status

18. Household income

AGE_DOB

SEX_ASK

SDC_COB

SDC_MRTL

INC_TOT

SSA_DPALL

19. Social support availability

20. Retirement status

21. Working

\section{RET_RTRD}

LBF_CURR

\section{GEN_DHDI}

GEN_DMHI

GEN_OWNAG

24. Healthy aging

SLS_DSCR

25. Life satisfaction

HWT_DBMI
"For some of the questions I'll be asking, I need to know your exact date of birth" and "What is your age?" (grouped: 45-54; 55-64; $65-74 ; 75-85)$

$M(I) / F(0)$

"In what country were you born?" (Code as Canada (I) or other (0))

"What is your current marital/partner status?" (Single/Married or common-law/Widowed/Divorced/Separated)

"What is your best estimate of the total household income received by all household members, from all sources, before taxes and deductions, in the past 12 months?" (<\$20k/\$20-\$49k/\$50-\$99k/\$100-\$149k/ $\$ 150 k+)$

This derived variable measures the overall level of functional social support that is available to the respondent. It includes all aspects asked about in the MOS Social Support Survey. Higher scores indicate higher levels of functional social support $(\mathrm{min}=0$, $\max =100)$

"At this time, do you consider yourself to be completely retired, partly retired or not retired?" (I (completely retired), 2 (partly retired), 3 (not retired))

"Are you currently working at a job or business?" (I (yes), 2 (no)) Health and Life Satisfaction Outcomes

This derived variable indicates the respondent's health status based on his/her own judgement. It is a recoded version of the questionnaire responses so that higher scores now indicate a more positively perceived health status. ( 1 - poor, 5 - excellent) This derived variable indicates the respondent's mental health status based on his/her own judgement. It is a recoded version of the questionnaire responses so that higher scores now indicate a more positively perceived mental health status. (I - poor, 5 excellent)

"In terms of your own healthy aging, would you say it is excellent, very good, good, fair, or poor?" (I - Excellent to 5 - Poor) This variable describes participants' satisfaction with life and is an aggregate score of the responses to the five items of the SWLS.
Individual responses to each item in the SWLS range from I - strongly disagree to 7 - strongly agree, and this score is a sum of those responses. Higher scores indicate a greater satisfaction with life. (min $5, \max 35)$

Body Mass Index

\section{Health Care Utilization}

\section{Emergency Depart-} ment HCU_EMEREG

"Have you been seen in an Emergency Department during the past 12 months?" (yes/no)

28. Admitted to Hospital

HCU_HLOVRNT

"Were you a patient in a hospital overnight during the past 12 months?" (yes/no)

29. Nursing Home

"Were you a patient in a nursing home or convalescent home during the past 12 months?" (yes/no) 